\title{
MOTION OF LEVEL SETS BY MEAN CURVATURE. I
}

\author{
L. C. EVANS \& J. SPRUCK
}

\begin{abstract}
We construct a unique weak solution of the nonlinear PDE which asserts each level set evolves in time according to its mean curvature. This weak solution allows us then to define for any compact set $\Gamma_{0}$ a unique generalized motion by mean curvature, existing for all time. We investigate the various geometric properties and pathologies of this evolution.
\end{abstract}

\section{Introduction}

We set forth in this paper rigorous justification of a new approach for defining and then investigating the evolution of a hypersurface in $\mathbb{R}^{n}$ moving according to its mean curvature. This problem has been long studied using parametric methods of differential geometry (see, for instance, Gage [15], [16], Gage-Hamilton [17], Grayson [19], Huisken [23], Ecker-Huisken [10], etc.). In this classical setup, we are given at time 0 a smooth hypersurface $\Gamma_{0}$ which is, say, the connected boundary of a bounded open subset of $\mathbb{R}^{n}$. As time progresses we allow the surface to evolve, by moving each point at a velocity equal to $(n-1)$ times the mean curvature vector at that point. Assuming this evolution is smooth, we define thereby for each $t>0$ a new hypersurface $\Gamma_{t}$. The primary problem is then to study geometric properties of $\left\{\Gamma_{t}\right\}_{t>0}$ in terms of $\Gamma_{0}$.

For the case $n=2$ this program has been successfully carried out in great detail (see [17], [19]). For $n \geq 3$, however, it is fairly clear that even if $\Gamma_{0}$ is smooth, a smooth evolution as envisioned above cannot exist beyond some initial time interval. Imagine, for instance, $\Gamma_{0}$ to be the boundary of a "dumbbell" shaped region in $\mathbb{R}^{3}$, as illustrated in Figure 1 (next page).

In view of Grayson [20] and numerical calculations of Sethian [35], we expect that as time evolves, the surface will smoothly evolve (and shrink)

Received August 14, 1989. Both authors were supported by National Science Foundation grants DMS-86-10730, DMS-86-01531 (L. C. Evans), and DMS-8501952 (J. Spruck). The second author was also supported in part by Department of Energy grant DE-FG0286ER250125. 


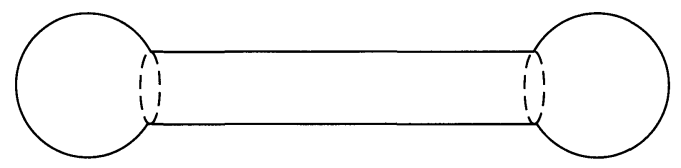

FIGURE 1

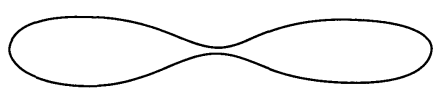

FIGURE 2

up until a critical time $t_{*}>0$ when the two ends pinch off, as drawn in Figure 2.

After this time, the classical motion via mean curvature is undefined. In addition, if it were possible to define the subsequent motion in some reasonable way, we expect $\Gamma_{t}$ for $t>t_{*}$ to comprise two pieces which pull apart at time $t_{*}$. If this were so, then $\Gamma_{t}$ would have changed topological type. This possibility suggests inherent problems in the classical differential geometric approach of regarding $\Gamma_{0}$ as a parametrized surface: the parametrization will in general develop singularities.

What is needed is an alternative description of the evolution for all times $t>0$, sufficiently general as to allow for the possible onset of singularities and attendant topological complications. To our knowledge there have been two different such undertakings, by Brakke [5] and by Osher-Sethian [33] (see also the note at the end of this section). Brakke [5] recasts the mean curvature motion problem (even in arbitrary codimension) into the setting of varifold theory from geometric measure theory (cf. Allard [2]). Brakke defines and then constructs an appropriate generalized varifold solution, which is defined for all time (although it may vanish after a finite time). He then deduces many geometric properties and under an additional density assumption establishes partial regularity. The principal drawback seems to be the lack of any uniqueness assertion.

A completely different viewpoint is to be found in the paper [33] by Osher and Sethian. Their approach, recast slightly, is this: given the initial hypersurface $\Gamma_{0}$ as above, select some continuous function $g: \mathbb{R}^{n} \rightarrow \mathbb{R}$ so that

$$
\Gamma_{0}=\left\{x \in \mathbb{R}^{n} \mid g(x)=0\right\} .
$$

Consider then the parabolic PDE

$$
u_{t}=\left(\delta_{i j}-u_{x_{i}} u_{x_{j}} /|D u|^{2}\right) u_{x_{i} x_{j}} \text { in } \mathbb{R}^{n} \times[0, \infty),
$$




$$
u=g \text { on } \mathbb{R}^{n} \times\{t=0\},
$$

for the unknown $u=u(x, t),\left(x \in \mathbb{R}^{n}, t \geq 0\right)$. Now the PDE (1.2) says that each level set of $u$ evolves according to its mean curvature, at least in regions where $u$ is smooth and its spacial gradient $D u$ does not vanish. Consequently, focusing our attention on the set $\{u=0\}$, it seems reasonable in view of $(1.1),(1.2)$ to define

$$
\Gamma_{t} \equiv\left\{x \in \mathbb{R}^{n} \mid u(x, t)=0\right\}
$$

for each time $t>0$. Osher and Sethian [33] and Sethian [35] introduce various techniques to study (1.2) and related PDE's numerically, thereby to track computationally the evolution of $\Gamma_{0}$ into $\Gamma_{t}(t \geq 0)$. (Notice by the way that our utilizing (1.1)-(1.3) amounts in the language of fluid mechanics to adopting an Eulerian viewpoint, as opposed to the Lagrangian, parametric viewpoint of classical differential geometry.)

Our purpose here is to provide theoretical justification for this approach. The undertaking is analytically subtle, principally because the mean curvature evolution equation (1.2) is nonlinear, degenerate, and indeed even undefined at points where $D u=0$. In addition, it is not so clear that our definition (1.3) is independent of the choice of initial function $g$ verifying (1.1). We will resolve these problems by introducing an appropriate definition of a weak solution for (1.2), inspired by the notion of so-called "viscosity solutions" of nonlinear PDE as in Evans [12], Crandall-Lions [9], Crandall-Evans-Lions [8], Lions [32], Jensen [25], and Ishii [24]. We then prove that there exists a unique weak solution of (1.2), and, further, that definition (1.3) is then independent of the choice of initial function $g$ satisfying (1.1). We additionally check that $\left\{\Gamma_{t}\right\}_{t \geq 0}$ so defined agrees with the classical notion of motion via mean curvature, over any time interval for which the latter exists. Finally we employ the PDE (1.2) to deduce assorted geometric properties of $\left\{\Gamma_{t}\right\}_{t \geq 0}$.

The main theoretical advantage of (1.1)-(1.3) as compared with Brakke's varifold methods seems to us to be the following uniqueness assertion: the set $\Gamma_{t}$ is unambiguously defined by (1.3) once we have a uniqueness assertion for the PDE (1.2). The primary disadvantage is that our techniques work only in codimension one.

In a companion paper [14] we give a new proof of short time existence for classical motion by mean curvature by studying the PDE solved by the distance function. We also hope to establish in a forthcoming paper a partial regularity theorem for $\left\{\Gamma_{t}\right\}_{t \geq 0}$.

Our paper is organized as follows. In $\S 2$ we motivate and introduce our definition of weak solution for (1.2) and in $\S 3$ we prove the uniqueness of 
a weak solution. $\S 4$ establishes the existence of a weak solution to (1.2). In $\S 5$ we verify the independence of the definition (1.3) on the choice of $g$. $\S 6$ contains a consistency check that the definition (1.3) agrees with the classical motion by mean curvature, if and so long as the latter exists. $\S \S 7$ and 8 contain various geometric assertions, examples of pathologies, and conjectures.

After this work was completed, we learned of the recent paper of Chen, Giga, and Goto [7], which announces results very similar to ours, especially the existence of a unique weak solution of the PDE (1.2), (1.3). Their work includes as well generalizations to other geometric problems.

Another new paper concerning curvature and viscosity solutions is Trudinger [36].

\section{Definition and elementary properties of weak solutions}

2.1. Heuristics. We start with a formal derivation of the mean curvature evolution PDE (1.2). For this, suppose temporarily $u=u(x, t)$ is a smooth function whose spatial gradient $D u=\left(u_{x_{1}}, \cdots, u_{x_{n}}\right)$ does not vanish in some open region $O$ of $\mathbb{R}^{n} \times(0, \infty)$. Assume further that each level set of $u$ smoothly evolves according to its mean curvature, as described in $\S 1$. We focus our attention onto any one such level set, and for definiteness consider the zero sets

$$
\Gamma_{t} \equiv\left\{x \in \mathbb{R}^{n} \mid u(x, t)=0\right\} \quad(t \geq 0) .
$$

Let $\nu=\nu(x, t)$ be a smooth unit normal vector field to $\left\{\Gamma_{t}\right\}_{t \geq 0}$ in $O$. Then

$$
-\frac{1}{n-1} \operatorname{div}(\nu) \nu
$$

is the mean curvature vector field. Thus if we fix $t \geq 0, x \in \Gamma_{t} \cap O$, the point $x$ evolves according to the nonautonomous ODE

$$
\left\{\begin{array}{l}
\dot{x}(s)=-[\operatorname{div}(\nu) \nu](x(s), s) \quad(s>t), \\
x(t)=x .
\end{array}\right.
$$

As $x(s) \in \Gamma_{s}(s \geq t)$, we have $u(x(s), s)=0 \quad(s>t)$; and so

$$
0=\frac{d}{d s} u(x(s), s)=-[(D u \cdot \nu) \operatorname{div}(\nu)](x(s), s)+u_{t}(x(s), s) .
$$

Setting $s=t$, we discover

$$
u_{t}=(D u \cdot \nu) \operatorname{div}(\nu) \text { at }(x, t)
$$


Choosing then

$$
\nu \equiv \frac{D u}{|D u|}
$$

it follows that

$$
u_{t}=|D u| \operatorname{div}\left(\frac{D u}{|D u|}\right)=\left(\delta_{i j}-\frac{u_{x_{i}} u_{x_{j}}}{|D u|^{2}}\right) u_{x_{i} x_{j}} \text { at }(x, t) .
$$

Similar reasoning demonstrates this PDE to hold throughout the region $O$.

Now, conversely, assume $u$ is a smooth solution of (2.4) in some region $O$ with $D u$ nonvanishing. Fix $t>0, x \in \Gamma_{t} \cap O$ and solve then the ODE (2.2), (2.3). Since $u$ solves (2.4), we deduce as above

$$
u(x(s), s)=0 \quad(s>t) .
$$

Consequently the zero sets, and similarly all the level sets, of $u$ evolve in $O$ according to their mean curvatures.

Since the motion of any level set thus depends only upon its own geometry, and not that of any other level set, our PDE (2.4) should be invariant under an arbitrary relabelling of these sets. Thus if $\Psi: \mathbb{R} \rightarrow \mathbb{R}$ is smooth, we expect that $v \equiv \Psi(u)$ will also be a solution of (2.4) in the region $O$. A direct calculation verifies this in the regions where $D v \neq 0$. Hence we see that an arbitrary function of a solution is still a solution; this is in strong contrast to the situation for uniformly parabolic PDE's. Indeed, we may informally interpret (2.4) as being somehow "uniformly parabolic along each level set", but as being also "totally degenerate across different level sets".

2.2. Weak solutions. The foregoing heuristics done with, we turn now to the full mean curvature evolution equation:

$$
\begin{gathered}
u_{t}=\left(\delta_{i j}-u_{x_{i}} u_{x_{j}} /|D u|^{2}\right) u_{x_{i} x_{j}} \text { in } \mathbb{R}^{n} \times(0, \infty), \\
u=g \text { on } \mathbb{R}^{n} \times\{t=0\}
\end{gathered}
$$

the function $g: \mathbb{R}^{n} \rightarrow \mathbb{R}$ being given. We want to define a notion of weak solution to (2.5). Since, however, the right-hand side of the PDE cannot be put into divergence form, we are not able to define a weak solution by means of formal integration by parts of derivatives onto a smooth test function (as for instance in Bombieri, De Giorgi, Giusti [4, §1]). We will instead follow Evans [12], Lions [32], Jensen [25], etc. and define our weak solution in terms of pointwise behavior with respect to a smooth test 
function. The primary difficulty will be to modify extant theory to cover the possibility that $D u$ may vanish.

Definition 2.1. A function $u \in C\left(\mathbb{R}^{n} \times[0, \infty)\right) \cap L^{\infty}\left(\mathbb{R}^{n} \times[0, \infty)\right)$ is a weak subsolution of $(2.5)$ provided that if

$$
u-\phi \text { has a local maximum at a point }\left(x_{0}, t_{0}\right) \in \mathbb{R}^{n} \times(0, \infty)
$$

for each $\phi \in C^{\infty}\left(\mathbb{R}^{n+1}\right)$, then

$$
\left\{\begin{array}{l}
\phi_{t} \leq\left(\delta_{i j}-\phi_{x_{i}} \phi_{x_{j}} /|D \phi|^{2}\right) \phi_{x_{i} x_{j}} \text { at }\left(x_{0}, t_{0}\right) \\
\text { if } D \phi\left(x_{0}, t_{0}\right) \neq 0
\end{array}\right.
$$

and

$$
\left\{\begin{array}{l}
\phi_{t} \leq\left(\delta_{i j}-\eta_{i} \eta_{j}\right) \phi_{x_{i} x_{j}} \text { at }\left(x_{0}, t_{0}\right) \\
\text { for some } \eta \in \mathbb{R}^{n} \text { with }|\eta| \leq 1, \text { if } D \phi\left(x_{0}, t_{0}\right)=0 .
\end{array}\right.
$$

Definition 2.2. A function $u \in C\left(\mathbb{R}^{n} \times[0, \infty)\right) \cap L^{\infty}\left(\mathbb{R}^{n} \times[0, \infty)\right)$ is a weak supersolution of $(2.5)$ provided that if

$$
u-\phi \text { has a local maximum at a point }\left(x_{0}, t_{0}\right) \in \mathbb{R}^{n} \times(0, \infty)
$$

for each $\phi \in C^{\infty}\left(\mathbb{R}^{n+1}\right)$, then

$$
\left\{\begin{array}{l}
\phi_{t} \geq\left(\delta_{i j}-\phi_{x_{i}} \phi_{x_{j}} /|D \phi|^{2}\right) \phi_{x_{i} x_{j}} \text { at }\left(x_{0}, t_{0}\right) \\
\text { if } D \phi\left(x_{0}, t_{0}\right) \neq 0
\end{array}\right.
$$

and

$$
\left\{\begin{array}{l}
\phi_{t} \geq\left(\delta_{i j}-\eta_{i} \eta_{j}\right) \phi_{x_{i} x_{j}} \text { at }\left(x_{0}, t_{0}\right) \\
\text { for some } \eta \in \mathbb{R}^{n} \text { with }|\eta| \neq 1, \text { if } D \phi\left(x_{0}, t_{0}\right)=0 .
\end{array}\right.
$$

Definition 2.3. A function $u \in C\left(\mathbb{R}^{n} \times[0, \infty)\right) \cap L^{\infty}\left(\mathbb{R}^{n} \times[0, \infty)\right)$ is a weak solution of $(2.5)$ provided $u$ is both a weak subsolution and a weak supersolution.

As preliminary motivation for these definitions, suppose $u$ is a smooth function on $\mathbb{R}^{n} \times(0, \infty)$ satisfying

$$
u_{t} \leq\left(\delta_{i j}-u_{x_{i}} u_{x_{j}} /|D u|^{2}\right) u_{x_{i} x_{j}}
$$

wherever $D u \neq 0$. Our function $u$ is thus a classical subsolution of $(2.5)$ on $\{D u \neq 0\}$. Suppose now $D u\left(x_{0}, t_{0}\right)=0$. Assume additionally there are points $\left(x_{k}, t_{k}\right) \rightarrow\left(x_{0}, t_{0}\right)$ for which $D u\left(x_{k}, t_{k}\right) \neq 0(k=1,2, \ldots)$. Then

$$
u_{t} \leq\left(\delta_{i j}-\eta_{j}^{k} \eta_{j}^{k}\right) u_{x_{i} x_{j}} \text { at }\left(x_{k}, t_{k}\right)
$$


for $\eta^{k} \equiv D u\left(x_{k}, t_{k}\right) /\left|D u\left(x_{k}, t_{k}\right)\right|$. Since $\left|\eta^{k}\right|=1 \quad(k=1,2, \ldots)$ we may as necessary pass to a subsequence so that $\eta^{k} \rightarrow \eta$ in $\mathbb{R}^{n},|\eta|=1$. Passing to limits above, we find

$$
u_{t} \leq\left(\delta_{i j}-\eta_{i} \eta_{j}\right) u_{x_{i} x_{j}} \text { at }\left(x_{0}, t_{0}\right)
$$

If, on the other hand, there do not exist such points $\left\{\left(x_{k}, t_{k}\right)\right\}_{k=1}^{\infty}$, then $D u=0$ near $\left(x_{0}, t_{0}\right)$, and so $D^{2} u=0$ and $u$ is a function of $t$ only, near $\left(x_{0}, t_{0}\right)$. Moving to the edge of the set $\{D u=0\}$, we see that $u$ is a nonincreasing function of $t$. Thus

$$
u_{t} \leq\left(\delta_{i j}-\eta_{i} \eta_{j}\right) u_{x_{i} x_{j}} \text { at }\left(x_{0}, t_{0}\right)
$$

for any $\eta \in \mathbb{R}^{n}$.

Further motivation for our definition of weak solution, and, in particular, an explanation as to why we assume only $|\eta| \leq 1$ in (2.9), (2.12), will be found in $\S 2.4$.

2.3. An equivalent definition. It will be convenient to have at hand certain alternative definitions. We write $z=(x, t), z_{0}=\left(x_{0}, t_{0}\right)$ and below implicitly sum $i, j$ from 1 to $n$.

Definition 2.4. A function $u \in C\left(\mathbb{R}^{n} \times[0, \infty)\right) \cap L^{\infty}\left(\mathbb{R}^{n} \times[0, \infty)\right)$ is a weak subsolution of $(2.5)$ if whenever $\left(x_{0}, t_{0}\right) \in \mathbb{R}^{n} \times(0, \infty)$ ard

$$
\begin{aligned}
u(x, t) \leq & u\left(x_{0}, t_{0}\right)+p \cdot\left(x-x_{0}\right)+q\left(t-t_{0}\right) \\
& +\frac{1}{2}\left(z-z_{0}\right)^{T} R\left(z-z_{0}\right)+o\left(\left|z-z_{0}\right|^{2}\right) \text { as } z \rightarrow z_{0}
\end{aligned}
$$

for some $p \in \mathbb{R}^{n}, q \in \mathbb{R}, R=\left(\left(r_{i j}\right)\right) \in S^{n+1 \times n+1}$, then

$$
q \leq\left(\delta_{i j}-p_{i} p_{j} /|p|^{2}\right) r_{i j} \text { if } p \neq 0
$$

and

$$
q \leq\left(\delta_{i j}-\eta_{i} \eta_{j}\right) r_{i j} \text { for some }|\eta| \leq 1 \text {, if } p=0 .
$$

Definition 2.5. A function $u \in C\left(\mathbb{R}^{n} \times[0, \infty)\right) \cap L^{\infty}\left(\mathbb{R}^{n} \times[0, \infty)\right)$ is a weak supersolution of $(2.5)$ if whenever $\left(x_{0}, t_{0}\right) \in \mathbb{R}^{n} \times(0, \infty)$ and

$$
\begin{aligned}
u(x, t) \geq & u\left(x_{0}, t_{0}\right)+p \cdot\left(x-x_{0}\right)+q\left(t-t_{0}\right) \\
& +\frac{1}{2}\left(z-z_{0}\right)^{T} R\left(z-z_{0}\right)+o\left(\left|z-z_{0}\right|^{2}\right) \quad \text { as } z \rightarrow z_{0}
\end{aligned}
$$

for some $p \in \mathbb{R}^{n}, q \in \mathbb{R}, R=\left(\left(r_{i j}\right)\right) \in S^{n+1 \times n+1}$, then

$$
q \geq\left(\delta_{i j}-p_{i} p_{j} /|p|^{2}\right) r_{i j} \quad \text { if } p \neq 0
$$


and

$$
q \geq\left(\delta_{i j}-\eta_{i} \eta_{j}\right) r_{i j} \text { for some }|\eta| \leq 1 \text {, if } p=0 \text {. }
$$

Theorem 2.6. Definitions 2.1 and 2.4 are equivalent, and Definitions 2.2 and 2.5 are equivalent.

This follows as in, for instance, Jensen [25], Ishii [24].

\subsection{Properties of weak solutions.}

Theorem 2.7. (i) Assume $u_{k}$ is a weak solution of (2.5) for $k=1,2, \ldots$ and $u_{k} \rightarrow u$ boundedly and locally uniformly on $\mathbb{R}^{n} \times[0, \infty)$. Then $u$ is a weak solution.

(ii) An analogous assertion holds for weak subsolutions and supersolutions.

Proof. 1. Choose $\phi \in C^{\infty}\left(\mathbb{R}^{n+1}\right)$ and suppose first $u-\phi$ has a strict local maximum at some point $\left(x_{0}, t_{0}\right) \in \mathbb{R}^{n} \times(0, \infty)$. As $u^{k} \rightarrow u$ uniformly near $\left(x_{0}, t_{0}\right)$,

(2.19) $u_{k}-\phi$ has a local maximum at a point $\left(x_{k}, t_{k}\right)(k=1,2, \ldots)$

with

$$
\left(x_{k}, t_{k}\right) \rightarrow\left(x_{0}, t_{0}\right) \text { as } k \rightarrow \infty .
$$

Since $u_{k}$ is a weak solution, we have either

$$
\phi_{t} \leq\left(\delta_{i j}-\phi_{x_{i}} \phi_{x_{i}} /|D \phi|^{2}\right) \phi_{x_{i} x_{j}} \text { at }\left(x_{k}, t_{k}\right)
$$

if $D \phi\left(x_{k}, t_{k}\right) \neq 0$, or

$$
\phi_{t} \leq\left(\delta_{i j}-\eta_{i}^{k} \eta_{j}^{k}\right) \phi_{x_{i} x_{j}} \text { at }\left(x_{k}, t_{k}\right)
$$

for some $\eta^{k} \in \mathbb{R}^{n}$ with $\left|\eta^{k}\right| \leq 1$, if $D \phi\left(x_{k}, t_{k}\right)=0$.

2. Assume first $D \phi\left(x_{0}, t_{0}\right) \neq 0$. Then $D \phi\left(x_{k}, t_{k}\right) \neq 0$ for all large enough $k$. Hence we may pass to limits in the equalities (2.21) to discover

$$
\phi_{t} \leq\left(\delta_{i j}-\phi_{x_{i}} \phi_{x_{j}} /|D \phi|^{2}\right) \text { at }\left(x_{0}, t_{0}\right) \text {. }
$$

3. Next, suppose $D \phi\left(x_{0}, t_{0}\right)=0$. We set

$$
\xi^{k} \equiv \begin{cases}(D \phi /|D \phi|)\left(x_{k}, t_{k}\right) & \text { if } D \phi\left(x_{k}, t_{k}\right) \neq 0, \\ \eta^{k} & \text { if } D \phi\left(x_{k}, t_{k}\right)=0 .\end{cases}
$$

Passing if necessary to a subsequence we may assume $\xi^{k} \rightarrow \eta$. Then $|\eta| \leq 1$. Utilizing now (2.22), we deduce as well

$$
\phi_{t} \leq\left(\delta_{i j}-\eta_{i} \eta_{j}\right) \phi_{x_{i} x_{j}} \text { at }\left(x_{0}, t_{0}\right)
$$


4. If $u-\phi$ has only a local maximum at $\left(x_{0}, t_{0}\right)$ we apply the above argument to

$$
\psi(x, t) \equiv \phi(x, t)+\left|x-x_{0}\right|^{4}+\left(t-t_{0}\right)^{4},
$$

so that $u-\psi$ has a strict local maximum at $\left(x_{0}, t_{0}\right)$. Hence $u$ is a weak subsolution. Similar reasoning verifies that $u$ is a weak supersolution as well.

Theorem 2.8. Assume $u$ is a weak solution of (2.5) and $\Psi: \mathbb{R} \rightarrow \mathbb{R}$ is continuous. Then $v \equiv \Psi(u)$ is a weak solution.

Proof. 1. Assume first $\Psi$ is smooth, with

$$
\Psi^{\prime}>0 \text { on } \mathbb{R} \text {. }
$$

Let $\phi \in C^{\infty}\left(\mathbb{R}^{n+1}\right)$ and suppose $v-\phi$ has a local maximum at $\left(x_{0}, t_{0}\right)$. Adding as necessary a constant to $\phi$, we may assume

$$
\left\{\begin{array}{l}
v\left(x_{0}, t_{0}\right)=\phi\left(x_{0}, t_{0}\right) \\
v(x, t) \leq \phi(x, t) \text { for all }(x, t) \text { near }\left(x_{0}, t_{0}\right) .
\end{array}\right.
$$

In view of (2.26), $\Phi \equiv \Psi^{-1}$ is defined and smooth near $u\left(x_{0}, t_{0}\right)$, with

$$
\Phi^{\prime}>0 \text {. }
$$

From (2.27) therefore we see

$$
\left\{\begin{array}{l}
u\left(x_{0}, t_{0}\right)=\psi\left(x_{0}, t_{0}\right) \\
u(x, t) \leq \psi(x, t) \text { for all }(x, t) \text { near }\left(x_{0}, t_{0}\right)
\end{array}\right.
$$

where

$$
\psi \equiv \Phi(\phi) .
$$

2. Since $u$ is a weak solution we conclude

$$
\psi_{t} \leq\left(\delta_{i j}-\psi_{x_{i}} \psi_{x_{j}} /|D \psi|^{2}\right) \psi_{x_{i} x_{j}} \text { at }\left(x_{0}, t_{0}\right)
$$

if $D \psi\left(x_{0}, t_{0}\right) \neq 0$, and

$$
\psi_{t} \leq\left(\delta_{i j}-\eta_{i} \eta_{j}\right) \psi_{x_{i} x_{j}} \text { at }\left(x_{0}, t_{0}\right)
$$

for some $|\eta| \leq 1$, if $D \psi\left(x_{0}, t_{0}\right)=0$. Now $D \phi\left(x_{0}, t_{0}\right)=0$ if and only if $D \psi\left(x_{0}, t_{0}\right)=0$. Consequently (2.31) is obtained if $D \phi\left(x_{0}, t_{0}\right) \neq 0$; in which case we substitute $(2.30)$ to compute

$$
\Phi^{\prime} \phi_{t} \leq\left(\delta_{i j}-\frac{\left(\Phi^{\prime}\right)^{2} \phi_{x_{i}} \phi_{x_{j}}}{\left(\Phi^{\prime}\right)^{2}|D \phi|^{2}}\right)\left(\Phi^{\prime} \phi_{x_{i} x_{j}}+\Phi^{\prime \prime} \phi_{x_{i}} \phi_{x_{j}}\right) \text { at }\left(x_{0}, t_{0}\right) \text {. }
$$


Since $\Phi^{\prime}>0$, we simplify and obtain

$$
\phi_{t} \leq\left(\delta_{i j}-\phi_{x_{i}} \phi_{x_{j}} /|D \phi|^{2}\right) \phi_{x_{i} x_{j}} \text { at }\left(x_{0}, t_{0}\right) \text {. }
$$

Suppose on the other hand $D \phi\left(x_{0}, t_{0}\right)=0$. Then (2.32) is valid for some $|\eta| \leq 1$. We substitute (2.30) and compute

$$
\Phi^{\prime} \phi_{t} \leq\left(\delta_{i j}-\eta_{i} \eta_{j}\right)\left(\Phi^{\prime} \phi_{x_{i} x_{j}}+\Phi^{\prime \prime} \phi_{x_{i}} \phi_{x_{j}}\right) \quad \text { at }\left(x_{0}, t_{0}\right) \text {. }
$$

Since $D \phi=0$, the term involving $\Phi^{\prime \prime}$ is zero. Thus

$$
\phi_{t} \leq\left(\delta_{i j}-\eta_{i} \eta_{j}\right) \phi_{x_{i} x_{j}} \text { at }\left(x_{0}, t_{0}\right) \text {. }
$$

We similarly have the opposite inequalities to (2.33), (2.34) should $v-\phi$ have a local minimum at $\left(x_{0}, t_{0}\right)$.

3. Now assume instead of $(2.20)$ that

$$
\Psi^{\prime}<0 \text { on } \mathbb{R} \text {. }
$$

Then $\Phi^{\prime}<0$ on $\mathbb{R}$ as well. Thus (2.27) now implies

$$
\left\{\begin{array}{l}
u\left(x_{0}, t_{0}\right)=\psi\left(x_{0}, t_{0}\right), \\
u(x, t) \geq \psi(x, t) \text { for all }(x, t) \text { near }\left(x_{0}, t_{0}\right) .
\end{array}\right.
$$

Since $u$ is a weak solution either

$$
\psi_{t} \geq\left(\delta_{i j}-\psi_{x_{i}} \psi_{x_{j}} /|D \psi|^{2}\right) \psi_{x_{i} x_{j}} \text { at }\left(x_{0}, t_{0}\right)
$$

if $D \psi\left(x_{0}, t_{0}\right) \neq 0$, or

$$
\psi_{t} \geq\left(\delta_{i j}-\eta_{i} \eta_{j}\right) \psi_{x_{i} x_{j}} \text { at }\left(x_{0}, t_{0}\right)
$$

for some $|\eta| \leq 1$,if $D \psi\left(x_{0}, t_{0}\right)=0$. Since now $\Phi^{\prime}<0$, we deduce as above either (2.33) or (2.34).

4. We have so far shown that $v=\Psi(u)$ is a weak solution provided $\Psi$ is smooth, with $\Psi^{\prime} \neq 0$. Approximating and using Theorem 2.7 we draw the same conclusion if $\Psi^{\prime} \geq 0$ or $\Psi^{\prime} \leq 0$ on $\mathbb{R}$.

5. Next assume $\Psi$ is smooth and there exist finitely many points $-\infty=$ $a_{0}<a_{1}<a_{2}<\cdots<a_{m}<a_{m+1}=+\infty$ such that

(2.36) $\Psi$ is monotone on the intervals $\left(a_{j}, a_{j+1}\right)(j=0, \cdots, m)$

and

(2.37) $\Psi$ is constant on the intervals $\left(a_{j}-\sigma, a_{j}+\sigma\right)(j=1, \cdots, m)$ for some $\sigma>0$.

Suppose $v-\phi$ has a maximum at $\left(x_{0}, t_{0}\right)$. Then

$$
u\left(x_{0}, t_{0}\right) \in\left(a_{j}-\sigma / 2, a_{j+1}+\sigma / 2\right) \text { for some } j \in\{0, \cdots, m\} \text {. }
$$


As $\Psi$ is monotone on $\left(a_{j}-\sigma, a_{j+1}+\sigma\right)$ and $u$ is continuous, we can apply steps $1-4$ in some neighborhood of $\left(x_{0}, t_{0}\right)$ to deduce (2.33) or (2.34). The reverse inequalities are similarly obtained if $v-\phi$ has a minimum.

6. Finally suppose only that $\Psi$ is continuous. We construct a sequence of smooth functions $\left\{\Psi^{k}\right\}_{k=1}^{\infty}$ each verifying the structural assumptions (2.36), (2.37) so that $\Psi^{k} \rightarrow \Psi$ uniformly on $\left[-\|u\|_{L^{\infty}},\|u\|_{L^{\infty}}\right]$. Hence

$$
v^{k}=\Psi^{k}(u) \rightarrow v \equiv \Psi(u)
$$

bounded and uniformly. Then Theorem 2.7 asserts $v$ to be a weak solution.

\section{Uniqueness and comparison of weak solutions}

3.1. Preliminaries. Our plan, as in Jensen [25] and Jensen-LionsSouganidis [26], is to regularize using sup and inf convolutions, defined as follows. Assume $w: \mathbb{R}^{n} \times[0, \infty) \rightarrow \mathbb{R}$ is continuous and bounded. If $\epsilon>0$, then we write

$$
\begin{aligned}
& w^{\epsilon}(x, t) \equiv \sup _{\substack{y \in \mathbb{R}^{n} \\
s \in[0, \infty)}}\left\{w(y, s)-\epsilon^{-1}\left(|x-y|^{2}+(t-s)^{2}\right)\right\}, \\
& w_{\epsilon}(x, t) \equiv \inf _{\substack{y \in \mathbb{R}^{n} \\
s \in[0, \infty)}}\left\{w(y, s)+\epsilon^{-1}\left(|x-y|^{2}+(t-s)^{2}\right)\right\},
\end{aligned}
$$

for $x \in \mathbb{R}^{n}, t \in[0, \infty)$. Note that since $w$ is continuous and bounded, the "sup" and "inf" above can be replaced by "max" and "min".

Lemma 3.1 (Properties of sup and inf convolutions). There exist constants $A, B, C$, depending only on $\|w\|_{L^{\infty}\left(R^{n} \times[0, \infty)\right)}$, such that for $\epsilon>0$ the following hold:

(i) $w_{\epsilon} \leq w \leq w^{\epsilon}$ on $\mathbb{R}^{n} \times[0, \infty)$.

(ii) $\left\|w^{\epsilon}, w_{\epsilon}\right\|_{L^{\infty}\left(R^{n} \times[0, \infty)\right)} \leq A$.

(iii) If $y \in \mathbb{R}^{n}, s \in[0, \infty)$, and $w^{\epsilon}(x, t)=w(y, x)-\epsilon^{-1}\left(|x-y|^{2}+\right.$ $\left.(t-s)^{2}\right)$, then

$$
|x-y|,|t-s| \leq C \epsilon^{1 / 2} \equiv \sigma(\epsilon)
$$

A similar assertion holds for $w_{\epsilon}$.

(iv) $w^{\epsilon}, w_{\epsilon} \rightarrow w$ as $\epsilon \rightarrow 0^{+}$, uniformly on compact subsets of $\mathbb{R}^{n} \times[0, \infty)$.

(v) $\operatorname{Lip}\left(w^{\epsilon}\right), \operatorname{Lip}\left(w_{\epsilon}\right) \leq B / \epsilon$. 
(vi) The mapping

$$
(x, t) \mapsto w^{\epsilon}(x, t)+\epsilon^{-1}\left(|x|^{2}+t^{2}\right)
$$

is convex, and the mapping

$$
(x, t) \mapsto w_{\epsilon}(x, t)-\epsilon^{-1}\left(|x|^{2}+t^{2}\right)
$$

is concave.

(vii) Assume $w$ is a weak solution of $(2.5)$ in $\mathbb{R}^{n} \times(0, \infty)$. Then $w^{\epsilon}$ is a weak subsolution on $\mathbb{R}^{n} \times(\sigma(\epsilon), \infty)$. Similarly, if $w$ is a weak supersolution of (2.5), $w_{\epsilon}$ is a weak supersolution.

(viii) The function $w^{\epsilon}$ is twice differentiable a.e. and satisfies

$$
w_{t}^{\epsilon} \leq\left(\delta_{i j}-w_{x_{i}}^{\epsilon} w_{x_{j}}^{\epsilon} /\left|D w^{\epsilon}\right|^{2}\right) w_{x_{i} x_{j}}^{\epsilon}
$$

at each point of twice differentiability in $\mathbb{R}^{n} \times(\sigma(\epsilon), \infty)$, where $D w^{\epsilon} \neq 0$. Similarly, $w_{\epsilon}$ is twice differentiable a.e. and satisfies

$$
w_{\epsilon t} \geq\left(\delta_{i j}-w_{\epsilon x_{i}} w_{\epsilon x_{j}} /\left|D w_{\epsilon}\right|^{2}\right) w_{\epsilon x_{i} x_{j}}
$$

at each point of twice differentiability in $\mathbb{R}^{n} \times(\sigma(\epsilon), \infty)$, where $D w_{\epsilon} \neq 0$.

Proof. 1. Assertions (i) and (ii) are clear from the definitions, for $A=\|w\|_{L^{\infty}\left(R^{n} \times[0, \infty)\right)}$. Statement (iii) follows from (ii), and then (iv) is a consequence of the uniform continuity of $w$ on compact sets. In light of estimate (3.3) we have (v) as well.

2. For each $y \in \mathbb{R}^{n}, s \in[0, \infty)$, the mapping

$$
(x, t) \mapsto w(y, s)-\epsilon^{-1}\left(|x-y|^{2}+(t-s)^{2}\right)+\epsilon^{-1}\left(|x|^{2}+t^{2}\right)
$$

is affine. Consequently

$$
\begin{aligned}
(x, t) \mapsto \sup _{\substack{y \in \mathbb{R}^{n} \\
s \in[0, \infty)}}\left[w(y, s)-\epsilon^{-1}\left(|x-y|^{2}+(t-s)^{2}\right)+\epsilon^{-1}\left(|x|^{2}+t^{2}\right)\right] \\
\quad=w^{\epsilon}(x, t)+\epsilon^{-1}\left(|x|^{2}+t^{2}\right)
\end{aligned}
$$

is convex, and ( $\mathrm{v})$ is proved.

3. Assume $\phi \in C^{\infty}\left(\mathbb{R}^{n+1}\right)$ and $w^{\epsilon}-\phi$ has a local maximum at a point $\left(x_{0}, t_{0}\right)$, with $t_{0}>\sigma(\epsilon)$. We then employ (3.3) to choose $\left(y_{0}, s_{0}\right) \in$ $\mathbb{R}^{n} \times(0, \infty)$ so that

$$
w^{\epsilon}\left(x_{0}, t_{0}\right)=w\left(y_{0}, s_{0}\right)-\epsilon^{-1}\left(\left|x_{0}-y_{0}\right|^{2}+\left(t_{0}-s_{0}\right)^{2}\right) .
$$

Set

$$
\psi(x, t) \equiv \phi\left(x+x_{0}-y_{0}, t+t_{0}-s_{0}\right) .
$$


Since $w^{\epsilon}-\phi$ has a local maximum at $\left(x_{0}, t_{0}\right)$ we compute

$$
\begin{array}{r}
w\left(y_{0}, s_{0}\right)-\epsilon^{-1}\left(\left|x_{0}-y_{0}\right|^{2}+\left(t_{0}-s_{0}\right)^{2}\right)-\phi\left(x_{0}, t_{0}\right) \\
=w^{\epsilon}\left(x_{0}, t_{0}\right)-\phi\left(x_{0}, t_{0}\right) \geq w^{\epsilon}(x, t)-\phi(x, t) \\
\geq w(y, s)-\epsilon^{-1}\left(|x-y|^{2}+(t-s)^{2}\right)-\phi(x, t)
\end{array}
$$

for all $(x, t)$ near $\left(x_{0}, t_{0}\right)$ and all $(y, s) \in \mathbb{R}^{n} \times[0, \infty)$. Fix $(y, s)$ close to $\left(y_{0}, s_{0}\right)$ and set $x=y+x_{0}-y_{0}, t=s+t_{0}-s_{0}$ as above, to discover

$$
w\left(y_{0}, s_{0}\right)-\phi\left(x_{0}, t_{0}\right) \geq w(y, s)-\phi\left(y+x_{0}-y_{0}, s+t_{0}-s_{0}\right) .
$$

Using (3.6) we rewrite this as

$$
w\left(y_{0}, s_{0}\right)-\psi\left(y_{0}, s_{0}\right) \geq w(y, s)-\psi(y, s)
$$

for all $(y, s)$ near $\left(y_{0}, s_{0}\right)$. Hence $w-\phi$ has a local maximum at $\left(y_{0}, s_{0}\right)$ and thus

$$
\psi_{t} \leq\left(\delta_{i j}-\psi_{x_{i}} \psi_{x_{i}} /|D \psi|^{2}\right) \psi_{x_{i} x_{j}} \text { at }\left(y_{0}, s_{0}\right)
$$

if $D \psi\left(y_{0}, s_{0}\right) \neq 0$, and

$$
\psi_{t} \leq\left(\delta_{i j}-\eta_{i} \eta_{j}\right) \psi_{x_{j} x_{j}} \text { at }\left(y_{0}, s_{0}\right)
$$

for some $|\eta| \leq 1$, if $D \psi\left(y_{0}, s_{0}\right)=0$. Since

$$
\begin{gathered}
D \psi\left(y_{0}, s_{0}\right)=D \phi\left(x_{0}, t_{0}\right), \psi_{t}\left(y_{0}, s_{0}\right)=\phi_{t}\left(x_{0}, t_{0}\right), \\
D^{2} \phi\left(y_{0}, s_{0}\right)=D^{2} \phi\left(x_{0}, t_{0}\right),
\end{gathered}
$$

we immediately obtain

$$
\phi_{t} \leq\left(\delta_{i j}-\phi_{x_{i}} \phi_{x_{j}} /|D \phi|^{2}\right) \phi_{x_{i} x_{j}} \text { at }\left(x_{0}, t_{0}\right)
$$

or

$$
\phi_{t} \leq\left(\delta_{i j}-\eta_{i} \eta_{j}\right) \phi_{x_{i} x_{j}} \text { at }\left(x_{0}, t_{0}\right)
$$

according as $D \phi\left(x_{0}, t_{0}\right)=0$ or not, and (vii) is proved.

4. Owing to (vi), $w^{\epsilon}(x, t)+\epsilon^{-1}\left(|x|^{2}+t^{2}\right)$ is convex in $(x, t)$ and so is twice differentiable a.e. according to a theorem of Alexandroff (see, e.g., Krylov [30, Appendix 2]). Thus $w^{\epsilon}$ is twice differentiable a.e. In view of (vii) and Theorem 2.6, (3.4) holds at points of twice differentiability, where $D w^{\epsilon} \neq 0$. Hence (viii) is proved.

3.2. Comparison principle, uniqueness. We establish now a comparison assertion for weak solutions of our mean curvature evolution PDE. Many of the key technical devices in the proof are taken from Jensen [25] and Ishii [24]. 
Theorem 3.2. Assume that $u$ is a weak subsolution and $v$ is a weak supersolution of (2.5). Suppose further

$$
u \leq v \quad \text { on } \mathbb{R}^{n} \times\{t=0\} .
$$

Finally assume

$$
\left\{\begin{array}{l}
u \text { and } v \text { are constant, with } u \leq v, \\
\text { on } \mathbb{R}^{n} \times[0, \infty) \cap\{|x|+t \geq R\}
\end{array}\right.
$$

for some constant $R \geq 0$. Then

$$
u \leq v \quad \text { on } \mathbb{R}^{n} \times[0, \infty) .
$$

In particular, a weak solution of (2.5), (2.6) is unique.

Proof. 1. Should (3.9) fail, then

$$
\max _{(x, t) \in R^{n} \times[0, \infty)}(u-v) \equiv a>0 ;
$$

and so for $\alpha>0$ small enough,

$$
\max _{(x, t) \in R^{n} \times[0, \infty)}(u-v-\alpha t) \geq a / 2>0 .
$$

According to (3.8) we have

$$
u^{\epsilon}=u, \quad v_{\epsilon}=v \quad \text { on }\{|x|+t \geq 2 R\}
$$

for all small $\epsilon>0$. Note further $u^{\epsilon} \rightarrow u$ and $v_{\epsilon} \rightarrow v$ uniformly. Consequently if we fix $\epsilon>0$ small enough,

$$
\max _{(x, t) \in R^{n} \times[0, \infty)}\left(u^{\epsilon}-v_{\epsilon}-\alpha t\right) \geq a / 4>0 .
$$

2. Given $\delta>0$ define for $x, y \in \mathbb{R}^{n}$ and $t, t+s \in[0, \infty)$

$$
\Phi(x, y, t, s) \equiv u^{\epsilon}(x+y, t+s)-v_{\epsilon}(x, t)-\alpha t-\delta^{-1}\left(|y|^{4}+s^{4}\right) \text {. }
$$

Owing to (3.12) we see

$$
\max _{(x, t),(x+y, t+s) \in R^{n} \times[0, \infty)} \Phi \geq a / 4>0 .
$$

Choose now $\left(x_{1}, t_{1}\right),\left(x_{1}+y_{1}, t_{1}+s_{1}\right) \in \mathbb{R}^{n} \times[0, \infty)$ so that

$$
\Phi\left(x_{1}, y_{1}, t_{1}, s_{1}\right)=\max _{(x, t),(x+y, t+s) \in R^{n} \times[0, \infty)} \Phi \text {. }
$$

Note in view of (3.11), (3.13) and Lemma 3.1(ii) that such points exist. Since $\Phi\left(x_{1}, y_{1}, t_{1}, s_{1}\right)>0,(3.13)$ implies

$$
\left|y_{1}\right|,\left|s_{1}\right| \leq C \delta^{1 / 4}
$$


3. We claim next that if $\epsilon, \delta>0$ are fixed small enough, we have

$$
t_{1}, t_{1}+s>\sigma(\epsilon)
$$

with $\sigma(\epsilon)$ defined in (3.3). Indeed if $t_{1} \leq \sigma(\epsilon)$, then

$$
\begin{aligned}
a / 4 & \leq \Phi\left(x_{1}, y_{1}, t_{1}, s_{1}\right) & & \\
& \leq u^{\epsilon}\left(x_{1}+y_{1}, t_{1}+s_{1}\right)-v_{\epsilon}\left(x_{1}, t_{1}\right) & & \\
& =u\left(x_{1}+y_{1}, t_{1}+s_{1}\right)-v\left(x_{1}, t_{1}\right)+o(1) & & \text { as } \epsilon \rightarrow 0 \\
& =u\left(x_{1}+y_{1}, s_{1}\right)-v\left(x_{1}, 0\right)+o(1) & & \text { as } \epsilon \rightarrow 0 \\
& =u\left(x_{1}, 0\right)-v\left(x_{1}, 0\right)+o(1) & & \text { as } \epsilon, \delta \rightarrow 0 \\
& \leq o(1) & & \text { as } \epsilon, \delta \rightarrow 0,
\end{aligned}
$$

where we employed Lemma 3.1(ii), (3.16), (3.7), and the continuity of $u, v$. This is a contradiction for $\epsilon, \delta>0$ small enough; whence $t_{1}>$ $\sigma(\epsilon)$. Owing to (3.16) we may as necessary adjust $\delta$ smaller to ensure (3.17). Hereafter in the proof, $\alpha, \epsilon, \delta>0$ are fixed.

According to Lemma 3.1(vii),

$$
u^{\epsilon} \text { is a weak subsolution of }(2.5) \text { near }\left(x_{1}+y_{1}, t_{1}+s_{1}\right)
$$

and

$$
v_{\epsilon} \text { is a weak supersolution of }(2.5) \text { near }\left(x_{1}, t_{1}\right) .
$$

4. We now demonstrate

$$
y_{1} \neq 0 \text {. }
$$

Assume for contradiction that in fact $y_{1}=0$. Then (3.13), (3.15) imply

$$
\begin{aligned}
& u^{\epsilon}\left(x_{1}, t_{1}+s_{1}\right)-v_{\epsilon}\left(x, t_{1}\right)-\alpha t_{1}-\delta^{-1} s_{1}^{4} \\
& \quad \geq u^{\epsilon}(x+y, t+s)-v_{\epsilon}(x, t)-\alpha t-\delta^{-1}\left(|y|^{4}+s^{4}\right)
\end{aligned}
$$

for all $(x, t),(x+y, t+s) \in \mathbb{R}^{n} \times[0, \infty)$. Put $x=x_{1}$ and $t=t_{1}$ as above, and simplify to obtain the inequality

$$
u^{\epsilon}\left(x_{1}+y, t_{1}+s\right) \leq u^{\epsilon}\left(x_{1}, t_{1}+s_{1}\right)+\delta^{-1}|y|^{4}+\delta^{-1}\left(s^{4}-s_{1}^{4}\right)
$$

for $\left(x_{1}+y, t_{1}+s\right) \in \mathbb{R}^{n} \times[0, \infty)$. Set $r=s-s_{1}$ and rewrite to find

$$
\begin{aligned}
u^{\epsilon}\left(x_{1}+y, t_{1}+s_{1}+r\right) \leq & u^{\epsilon}\left(x_{1}, t_{1}+s_{1}\right)+4 s_{1}^{3} r / \delta+6 s_{1}^{2} r^{2} / \delta \\
& +O\left(|r|^{3}+|y|^{4}\right) \text { as }(y, r) \rightarrow(0,0) .
\end{aligned}
$$


Since $u^{\epsilon}$ is a weak subsolution near $\left(x_{1}+y_{1}, t_{1}+s_{1}\right)=\left(x_{1}, t_{1}+s_{1}\right)$, we may invoke (2.13), (2.15) with $x_{0}=x_{1}, t_{0}=t_{1}+s_{1}, p=0, q=4 s_{1}^{3} / \delta$, $r_{n+1, n+1}=12 s_{1}^{2} / \delta, r_{i j}=0$ otherwise. This gives

$$
4 s_{1}^{3} / \delta \leq 0 \text {. }
$$

Now go back and insert $y=x_{1}-x$ and $s=t_{1}+s_{1}-t$ into (3.21). This yields after simplifications:

$$
\begin{aligned}
v_{\epsilon}(x, t) \geq & v_{\epsilon}\left(x_{1}, t_{1}\right)+\left(4 s_{1}^{3} / \delta-\alpha\right)\left(t-t_{1}\right)-6 s_{1}^{2}\left(t-t_{1}\right)^{2} / \delta \\
& +O\left(\left|x-x_{1}\right|^{4}+\left|t-t_{1}\right|^{3}\right) \quad \text { as }(x, t) \rightarrow\left(x_{1}, t_{1}\right) .
\end{aligned}
$$

Now $v_{\epsilon}$ is a weak supersolution near $\left(x_{1}, t_{1}\right)$. Thus $(2.16),(2.18)$ with $x_{0}=x_{1}, t_{0}=t_{1}, p=0, q=4 s_{1}^{3} / \delta-\alpha, r_{n+1, n+1}=-12 s_{1}^{2} / \delta$, and $r_{i j}=0$ otherwise, imply

$$
4 s_{1}^{3} / \delta-\alpha \geq 0 \text {. }
$$

But now we have a contradiction with (3.22), since $\alpha>0$. This establishes (3.20).

5. Note next that in general if $f: \mathbb{R}^{m} \rightarrow \mathbb{R}$ is convex, then so is the mapping $(w, z) \mapsto f(w+z)$ on $\mathbb{R}^{2 m}$. Consequently Lemma 3.1(vi) asserts

$$
(x, y, t, s) \mapsto u^{\epsilon}(x+y, t+s)+\epsilon^{-1}\left(|x-y|^{2}+(t+s)^{2}\right)
$$

is convex. As

$$
(x, t) \mapsto-v_{\epsilon}(x, t)+\epsilon^{-1}\left(|x|^{2}+t^{2}\right)
$$

is convex as well, we see that

$$
(x, y, t, s) \mapsto \Phi(x, y, t, s)+C\left(|x|^{2}+|y|^{2}+t^{2}+s^{2}\right)
$$

is convex near $\left(x_{1}, y_{1}, t_{1}, s_{1}\right)$, for some sufficiently large constant $C=$ $C(\epsilon, \delta)$. Since $\Phi$ additionally attains its maximum at $\left(x_{1}, y_{1}, t_{1}, s_{1}\right)$ we may invoke Jensen [25]: there exist points $\left\{\left(x^{k}, y^{k}, t^{k}, s^{k}\right)\right\}_{k=1}^{\infty}$ such that

$$
\left(x^{k}, y^{k}, t^{k}, s^{k}\right) \rightarrow\left(x_{1}, y_{1}, t_{1}, s_{1}\right),
$$

$\Phi, u^{\epsilon}$ and $v_{\epsilon}$ are each twice differentiable

$$
\text { at }\left(x^{k}, y^{k}, t^{k}, s^{k}\right)(k=1,2, \ldots) \text {, }
$$

$$
\begin{gathered}
D_{x, y, t, s} \Phi\left(x^{k}, y^{k}, t^{k}, s^{k}\right) \rightarrow 0, \\
D_{x, y, t, s}^{2} \Phi\left(x^{k}, y^{k}, t^{k}, s^{k}\right) \leq o(1) I_{2 n+2} \quad \text { as } k \rightarrow \infty .
\end{gathered}
$$


6. Using (3.13), (3.25), we see

$$
\begin{aligned}
D_{x} \Phi\left(x^{k}, y^{k}, t^{k}, s^{k}\right) & =D u^{\epsilon}\left(x^{k}+y^{k}, t^{k}+s^{k}\right)-D v_{\epsilon}\left(x^{k}, t^{k}\right) \\
& \equiv p^{k}-\bar{p}^{k}, \\
D_{y} \Phi\left(x^{k}, y^{k}, t^{k}, s^{k}\right) & =D u^{\epsilon}\left(x^{k}+y^{k}, t^{k}+s^{k}\right)-4\left|y^{k}\right|^{2} y^{k} / \delta \\
& =p^{k}-4\left|y^{k}\right|^{2} y^{k} / \delta .
\end{aligned}
$$

Since $y^{k} \rightarrow y_{1}$, we deduce from (3.26) that

$$
p^{k}, \vec{p}^{k} \rightarrow 4\left|y_{1}\right|^{2} y_{1} / \delta \equiv p \quad \text { in } \mathbb{R}^{n} .
$$

Assertion (3.20) tells us $p \neq 0$ and so $p^{k}, \bar{p}^{k} \neq 0$ for large enough $k$.

Again employing (3.13), (3.26) we note

$$
\begin{aligned}
\Phi_{t}\left(x^{k}, y^{k}, t^{k}, s^{k}\right) & =u_{t}^{\epsilon}\left(x^{k}+y^{k}, t^{k}+s^{k}\right)-v_{\epsilon t}\left(x^{k}, t^{k}\right)-\alpha \\
& \equiv q^{k}-\bar{q}^{k}-\alpha .
\end{aligned}
$$

As $u^{\epsilon}$ and $v_{\epsilon}$ are Lipschitz, we may assume, upon passing to a subsequence and reindexing if necessary, that

$$
q^{k} \rightarrow q, \bar{q}^{k} \rightarrow \bar{q} \text { in } \mathbb{R} .
$$

Then (3.26) and (3.31) ensure

$$
q-\bar{q}=\alpha>0 .
$$

7. Next, (3.13) and (3.25) imply

$$
\begin{aligned}
D_{x}^{2} \Phi\left(x^{k}, y^{k}, t^{k}, s^{k}\right) & =D^{2} u^{\epsilon}\left(x^{k}+y^{k}, t^{k}+s^{k}\right)-D^{2} v_{\epsilon}\left(x^{k}, t^{k}\right) \\
& \equiv R^{k}-\bar{R}^{k}
\end{aligned}
$$

Now (3.27) forces

$$
R^{k}-\bar{R}^{k} \leq \epsilon_{k} I_{n},
$$

where $\epsilon_{k} \rightarrow 0$. Furthermore, Lemma 3.1(vi) shows $R^{k} \geq-C I_{n}$ and $\bar{R}^{k} \leq C I_{n}$, for $C=C(\epsilon)$. Thus

$$
-C I_{n} \leq R^{k} \leq \bar{R}^{k}+\epsilon_{k} I_{n} \leq C I_{n} .
$$

We may consequently suppose, passing as necessary to subsequences, that

$$
R^{k} \rightarrow R, \bar{R}^{k} \rightarrow \bar{R} \text { in } S^{n \times n},
$$


with

$$
R \leq \bar{R}
$$

8. Now recall (3.25) holds and $p^{k} \equiv D u^{\epsilon}\left(x^{k}+y^{k}, t^{k}+s^{k}\right), \bar{p}^{k} \equiv$ $D v_{\epsilon}\left(x^{k}, t^{k}\right)$ are nonzero for large $k$. Since $u^{\epsilon}$ is a weak subsolution near $\left(x_{1}+y_{1}, t_{1}+s_{1}\right)$ and $v_{\epsilon}$ is a weak supersolution near $\left(x_{1}, t_{1}\right)$, we thus have

$$
q^{k} \leq\left(\delta_{i j}-p_{i}^{k} p_{j}^{k} /\left|p^{k}\right|^{2}\right) r_{i j}^{k} \quad \text { and } \quad \bar{q}^{k} \geq\left(\delta_{i j}-\bar{p}_{i}^{k} \bar{p}_{j}^{k} /\left|\bar{p}^{k}\right|^{2}\right) \bar{r}_{i j}^{k}
$$

for all large $k$. We send $k$ to infinity, recalling (3.30), (3.32), and (3.36) to obtain

$$
q \leq\left(\delta_{i j}-p_{i} p_{j} /|p|^{2}\right) r_{i j} \text { and } \bar{q} \geq\left(\delta_{i j}-p_{i} p_{j} /|p|^{2}\right) \bar{r}_{i j},
$$

and, by subtracting,

$$
q-\bar{q} \leq\left(\delta_{i j}-p_{i} p_{j} /|p|^{2}\right)\left(r_{i j}-\bar{r}_{i j}\right) .
$$

Now the matrix $\left(\left(\delta_{i j}-p_{i} p_{j} /|p|^{2}\right)\right)$ is nonnegative and $R-\bar{R}$ is nonpositive, by (3.37). Consequently $q-\bar{q} \leq 0$, a contradiction to (3.33).

3.3. Contraction property.

Theorem 3.3. Assume that $u$ and $v$ are weak solutions of (2.5), such that

$$
u \text { and } v \text { are constant on } \mathbb{R}^{n} \times[0, \infty) \cap\{|x|+t \geq R\}
$$

for some constant $R>0$. Then

$$
\max _{0 \leq t<\infty}\|u(\cdot, t)-v(\cdot, t)\|_{L^{\infty}\left(R^{n}\right)}=\|u(\cdot, 0) 0-v(\cdot, 0)\|_{L^{\infty}\left(R^{n}\right)} \cdot
$$

Proof. Should (3.39) fail, we may assume

$$
\max _{(x, t) \in R^{n} \times[0, \infty)}(u-v) \equiv a>\|u(\cdot, 0)-v(\cdot, 0)\|_{L^{\infty}\left(R^{n}\right)} \equiv b .
$$

Then as in the proof of Theorem 3.2 as above, there exist $\alpha, \epsilon, \delta>0$ such that $\max _{(x, t),(x+y, t+s) \in R^{n} \times[0, \infty)} \Phi>b$, where $\Phi$ is defined by (3.13). We find a point $\left(x_{1}, y_{1}, t_{1}, s_{1}\right)$ satisfying (3.15) and check (3.17) is valid provided $\epsilon, \delta>0$ are small enough. The rest of the proof follows from that for Theorem 3.2.

\section{Existence of weak solutions}

4.1. Approximation; geometric interpretation. We turn our attention now to constructing a weak solution of the initial value problem (2.5), (2.6). We will assume that

$g$ is constant on $\left\{\mathbb{R}^{n}\right\} \cap\{|x| \geq S\}$ 
for some constant $S>0$ and additionally, for the moment at least, $g$ is smooth.

Our intention is to approximate (2.5), (2.6) by the PDE

$$
\begin{gathered}
u_{t}^{\epsilon}=\left(\delta_{i j}-\frac{u_{x_{i}}^{\epsilon} u_{x_{j}}^{\epsilon}}{\left|D u^{\epsilon}\right|^{2}+\epsilon^{2}}\right) u_{x_{i} x_{j}}^{\epsilon} \text { in } \mathbb{R}^{n} \times(0, \infty), \\
u^{\epsilon}=g \text { on } \mathbb{R}^{n} \times\{t=0\},
\end{gathered}
$$

for $0<\epsilon<1$. (The superscript $\epsilon$ here and hereafter is only a label and does not mean the sup-convolution (3.1).)

We interpret (4.2), (4.3) geometrically as follows. Assuming for the moment $u^{\epsilon}=u^{\epsilon}(x, t)$ to be a smooth solution of (4.2), (4.3), write $y=$ $\left(x, x_{n+1}\right) \in \mathbb{R}^{n+1}$ and define

$$
v^{\epsilon}(y, t) \equiv u^{\epsilon}(x, t)-\epsilon x_{n+1} .
$$

Then $\left|D_{y} v^{\epsilon}\right|^{2}=\left|D u^{\epsilon}\right|^{2}+\epsilon^{2}$, and thus our PDE (4.2) becomes

$$
\begin{gathered}
v_{t}^{\epsilon}=\left(\delta_{i j}-v_{y_{i}}^{\epsilon} v_{y_{j}}^{\epsilon} /\left|D v^{\epsilon}\right|^{2}\right) v_{y_{i} y_{j}}^{\epsilon} \text { in } \mathbb{R}^{n+1} \times[0, \infty), \\
v^{\epsilon}=g^{\epsilon} \text { on } \mathbb{R}^{n+1} \times\{t=0\},
\end{gathered}
$$

for $g^{\epsilon}(y) \equiv g(x)-\epsilon x_{n+1}$. As noted in $\S 2$, the PDE (4.5) says that each level set of $v^{\epsilon}$ evolves according to its mean curvature. This is, in particular, the case for the zero level sets

$$
\Gamma_{t}^{\epsilon} \equiv\left\{y \in \mathbb{R}^{n+1} \mid v^{\epsilon}(y, t)=0\right\} .
$$

But according to (4.4) each $\Gamma_{t}^{\epsilon}$ is a graph:

$$
\Gamma_{t}^{\epsilon}=\left\{y=\left(x, x_{n+1}\right) \in \mathbb{R}^{n+1} \mid x_{n+1}=\epsilon^{-1} u^{\epsilon}(x, t)\right\},
$$

and Ecker and Huisken [10] have shown the evolution of an entire graph by mean curvature remains a smooth entire graph for all time.

Geometrically, if as in $\S 1$ we are given $\Gamma_{0}$ as the boundary of a smooth, bounded, simply connected open set $U$ in $\mathbb{R}^{n}$, we select a smooth function $g$ with $g=0$ on $\Gamma_{0}, g<0$ in $U, g>0$ in $\mathbb{R}^{n}-\bar{U}$. Then $\Gamma_{0}^{\epsilon} \subset \mathbb{R}^{n+1}$ is the graph $\left\{x_{n+1}=\epsilon^{-1} g(x)\right\}$ as drawn in Figure 3 (next page).

For small $\epsilon, \Gamma_{0}^{\epsilon}$ roughly approximates the cylinder $\Gamma_{0} \times \mathbb{R}$. We may thus hope that for moderate $t>0$ and small $\epsilon>0$, the smooth graph $\Gamma_{t}^{\epsilon}$ will be close to the cylinder $\Gamma_{t} \times \mathbb{R}, \Gamma_{t}$ denoting the evolution of $\Gamma_{0}$ via its mean curvature in $\mathbb{R}^{n}$ (see Figure 4 ). 


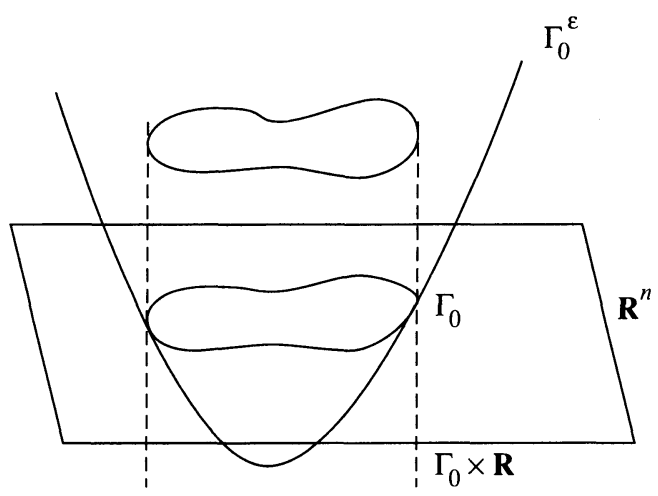

FIGURE 3

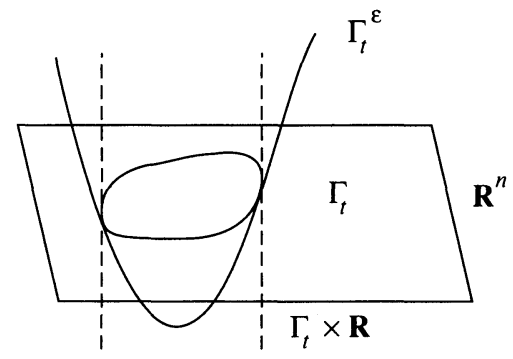

FIGURE 4

The idea then is that the complicated, possibly singular behavior of $\left\{\Gamma_{t}\right\}_{t \geq 0}$ in $\mathbb{R}^{n}$ will be approximated by the smooth evolution $\left\{\Gamma_{t}^{\epsilon}\right\}_{t \geq 0}$ in $\mathbb{R}^{n+1}$; in the sense that for a given $t>0, \Gamma_{t}^{\epsilon} \approx \Gamma_{t} \times \mathbb{R}$ if $\epsilon>0$ is very small. The illustrations provided make this expectation appear plausible, although there are a number of subtleties.

4.2. Solution of the approximate equations. We now investigate the approximations (4.2), (4.3) analytically.

Theorem 4.1. (i) For each $0<\epsilon<1$ there exists a unique smooth, bounded solution $u^{\epsilon}$ of (4.2), (4.3).

(ii) Additionally,

$$
\sup _{0<\epsilon<1}\left\|u^{\epsilon}, D u^{\epsilon}, u_{t}^{\epsilon}\right\|_{L^{\infty}\left(R^{n} \times[0, \infty)\right)} \leq C\|g\|_{C^{1,1}\left(R^{n}\right)} .
$$

Proof. 1. For each $0<\sigma<1$, consider the PDE

$$
\begin{gathered}
u_{t}^{\epsilon, \sigma}=a_{i j}^{\epsilon, \sigma}\left(D u^{\epsilon, \sigma}\right) u_{x_{i} x_{j}}^{\epsilon \sigma} \text { in } \mathbb{R}^{n} \times[0, \infty), \\
u^{\epsilon, \sigma}=g \quad \text { on } \mathbb{R}^{n} \times\{t=0\},
\end{gathered}
$$


for

$$
a_{i j}^{\epsilon, \sigma}(p) \equiv(1+\sigma) \delta_{i j}-\frac{p_{i} p_{j}}{|p|^{2}+\epsilon^{2}} \quad\left(p \in \mathbb{R}^{n}, 1 \leq i, j \leq n\right) .
$$

The smooth bounded coefficients $\left\{a_{i j}\right\}$ satisfy also the uniform ellipticity condition

$$
\sigma|\xi|^{2} \leq a_{i j}^{\epsilon, \sigma}(p) \xi_{i} \xi_{j} \quad\left(\xi \in \mathbb{R}^{n}\right)
$$

for each $p \in \mathbb{R}^{n}$, and consequently classical PDE theory gives the existence of a unique smooth bounded solution $u^{\epsilon, \sigma}$ (see, e.g., Ladyzhenskaja, Solonnikov, and Ural'tseva [31]). By the maximum principle,

$$
\left\|u^{\epsilon, \sigma}\right\|_{L^{\infty}\left(R^{n} \times[0, \infty)\right)}=\|g\|_{L^{\infty}\left(R^{n}\right)} .
$$

2. Now differentiate (4.8) with respect to $x_{l}$ :

$$
u_{x_{l t}}^{\epsilon, \sigma}=a_{i j}^{\epsilon, \sigma}\left(D u^{\epsilon, \sigma}\right) u_{x_{l} x_{i} x_{j}}^{\epsilon, \sigma}+a_{i j, p_{k}}^{\epsilon, \sigma}\left(D u^{\epsilon, \sigma}\right) u_{x_{l} x_{k}}^{\epsilon, \sigma} u_{x_{i} x_{j}}^{\epsilon, \sigma} .
$$

The maximum principle then implies

$$
\left\|D u^{\epsilon, \sigma}\right\|_{L^{\infty}\left(R^{n} \times[0, \infty)\right)}=\|D g\|_{L^{\infty}\left(R^{n}\right)} .
$$

Similarly

$$
\left\|u_{t}^{\epsilon, \sigma}\right\|_{L^{\infty}\left(R^{n} \times[0, \infty)\right)}=\left\|u_{t}^{\epsilon, \sigma}(\cdot, 0)\right\|_{L^{\infty}\left(R^{n}\right)} \leq C\left\|D^{2} g\right\|_{L^{\infty}\left(R^{n}\right)} .
$$

3. Since

$$
\left(1-\frac{L^{2}}{L^{2}+\epsilon^{2}}\right)|\xi|^{2} \leq a_{i j}^{\epsilon, \sigma}(p) \xi_{i} \xi_{j} \quad\left(\xi \in \mathbb{R}^{n}\right)
$$

provided $|p| \leq L$, we deduce from (4.10)-(4.12) and classical estimates that we have bounds, uniform in $0<\sigma<1$, on the derivatives of all orders of $\left\{u^{\epsilon, \sigma}\right\}_{0<\sigma<1}$. Consequently, uniqueness of the limit implies for each multi-index $\alpha$,

$$
D^{\alpha} u^{\epsilon, \sigma} \rightarrow D^{\alpha} u^{\epsilon} \quad \text { locally uniformly as } \sigma \rightarrow 0,
$$

for a smooth function $u^{\epsilon}$ solving (4.2), (4.3). Estimate (4.7) follows from (4.10)-(4.12).

4.3. Passage to limits.

Theorem 4.2. Assume $g: \mathbb{R}^{n} \rightarrow \mathbb{R}$ is continuous and satisfies (4.1). Then there exists a weak solution $u$ of (2.5), (2.6), such that

$$
u \text { is constant on } \mathbb{R}^{n} \times[0, \infty) \cap\{|x|+t \geq R\}
$$

for some $R>0$, depending only on the constant $S$ from (4.1).

Proof. 1. Suppose temporarily $g$ is smooth. Employing estimate (4.7) we can extract a subsequence $\left\{u^{\epsilon_{k}}\right\}_{k=1}^{\infty} \subset\left\{u^{\epsilon}\right\}_{0<\epsilon \leq 1}$ so that $\epsilon_{k} \rightarrow 0$ and 
$u^{\epsilon_{k}} \rightarrow u$ locally uniformly in $\mathbb{R}^{n} \times[0, \infty)$, for some bounded, Lipschitz function $u$.

2. We assert now that $u$ is a weak solution of (2.5), (2.6). For this, let $\phi \in C^{\infty}\left(\mathbb{R}^{n+1}\right)$ and suppose $u-\phi$ has a strict local maximum at a point $\left(x_{0}, t_{0}\right) \in \mathbb{R}^{n} \times(0, \infty)$. As $u^{\epsilon_{k}} \rightarrow u$ uniformly near $\left(x_{0}, t_{0}\right), u^{\epsilon_{k}}-\phi$ has a local maximum at a point $\left(x_{k}, t_{k}\right)$, with

$$
\left(x_{k}, t_{k}\right) \rightarrow\left(x_{0}, t_{0}\right) \text { as } k \rightarrow \infty .
$$

Since $u^{\epsilon_{k}}$ and $\phi$ are smooth, we have

$$
D u^{\epsilon_{k}}=D \phi, \quad u_{t}^{\epsilon_{k}}=\phi_{t}, \quad D^{2} u^{\epsilon_{k}} \leq D^{2} \phi \quad \text { at }\left(x_{k}, t_{k}\right) .
$$

Thus (4.2) implies

$$
\phi_{t}-\left(\delta_{i j}-\frac{\phi_{x_{i}} \phi_{x_{j}}}{|D \phi|^{2}+\epsilon_{k}^{2}}\right) \phi_{x_{i} x_{j}} \leq 0 \text { at }\left(x_{k}, t_{k}\right) .
$$

Suppose first $D \phi\left(x_{0}, t_{0}\right) \neq 0$. Then $D \phi\left(x_{k}, t_{k}\right) \neq 0$ for large $k$. We consequently may pass to limits in (4.15), recalling (4.14) to deduce

$$
\phi_{t} \leq\left(\delta_{i j}-\phi_{x_{i}} \phi_{x_{j}} /|D \phi|^{2}\right) \phi_{x_{i} x_{j}} \text { at }\left(x_{0}, t_{0}\right) \text {. }
$$

Next, assume instead $D \phi\left(x_{0}, t_{0}\right)=0$. Set

$$
\eta^{k} \equiv \frac{D \phi\left(x_{k}, t_{k}\right)}{\left(\left|D \phi\left(x_{k}, t_{k}\right)\right|^{2}+\epsilon_{k}^{2}\right)^{1 / 2}}
$$

so that $(4.15)$ becomes

$$
\phi_{t} \leq\left(\delta_{i j}-\eta_{i}^{k} \eta_{j}^{k}\right) \phi_{x_{i} x_{j}} \text { at }\left(x_{k}, t_{k}\right) .
$$

Since $\left|\eta^{k}\right| \leq 1$, we may assume, upon passing to a subsequence and reindexing if necessary, that $\eta^{k} \rightarrow \eta$ in $\mathbb{R}^{n}$ for some $|\eta| \leq 1$. Sending $k$ to infinity in (4.17), we discover

$$
\phi_{t} \leq\left(\delta_{i j}-\eta_{i} \eta_{j}\right) \phi_{x_{i} x_{j}} \text { at }\left(x_{0}, t_{0}\right) .
$$

If $u-\phi$ has a local maximum, but not necessarily a strict local maximum at $\left(x_{0}, t_{0}\right)$, we repeat the argument above with $\phi(x, t)$ replaced by

$$
\tilde{\phi}(x, t)=\phi(x, t)+\left|x-x_{0}\right|^{4}+\left(t-t_{0}\right)^{4},
$$

again to obtain (4.16) or (4.18).

Consequently, $u$ is a weak subsolution. That $u$ is a weak supersolution follows analogously. 
3. Finally we verify $u$ satisfies (4.13). Upon rescaling as necessary, we may as well assume

$$
|g| \leq 1 \quad \text { on } \mathbb{R}^{n}, \quad g=0 \quad \text { on } \mathbb{R}^{n} \cap\{|x| \geq 1\} .
$$

Consider now the auxiliary function (cf. Brakke [5, p. 25])

$$
v(x, t) \equiv \Psi\left(|x|^{2} / 2+(n-1) t\right) \quad\left(x \in \mathbb{R}^{n}, t>0\right),
$$

for

$$
\Psi(s) \equiv \begin{cases}0 & (s \geq 2) \\ (s-2)^{3} & (0 \leq s \leq 2) .\end{cases}
$$

Then for $\Psi \in C^{2}([0, \infty))$,

$$
\Psi^{\prime}(s)=\left\{\begin{array}{ll}
0 & (s \geq 2), \\
3(s-2)^{2} & (0 \leq s \leq 2),
\end{array} \quad \Psi^{\prime \prime}(s)= \begin{cases}0 & (s \geq 2), \\
6(s-2) & (0 \leq s \leq 2) .\end{cases}\right.
$$

In particular,

$$
\left|\Psi^{\prime \prime}(s)\right| \leq C\left(\Psi^{\prime}(s)\right)^{1 / 2} \quad(s \geq 0) .
$$

Now

$$
\begin{aligned}
v_{t}- & \left(\delta_{i j}-\frac{v_{x_{i}} v_{x_{i}}}{|D v|^{2}+\epsilon^{2}}\right) v_{x_{i} x_{j}} \\
= & (n-1) \Psi^{\prime}-\left(\delta_{i j}-\frac{\left(\Psi^{\prime}\right)^{2} x_{i} x_{j}}{\left(\Psi^{\prime}\right)^{2}|x|^{2}+\epsilon^{2}}\right)\left(\Psi^{\prime} \delta_{i j}+\Psi^{\prime \prime} x_{i} x_{j}\right) \\
= & \Psi^{\prime}\left[(n-1)-\left(\delta_{i j}-\frac{\left(\Psi^{\prime}\right)^{2} x_{i} x_{j}}{\left(\Psi^{\prime 2}|x|^{2}+\epsilon^{2}\right.}\right) \delta_{i j}\right] \\
& -\Psi^{\prime \prime}\left[\left(\delta_{i j}-\frac{\left(\Psi^{\prime}\right)^{2} x_{i} x_{j}}{\left(\Psi^{\prime}\right)^{2}|x|^{2}+\epsilon^{2}}\right) x_{i} x_{j}\right] \\
\equiv & A+B .
\end{aligned}
$$

We further compute

$$
A=-\Psi^{\prime} \frac{\epsilon^{2}}{\left(\Psi^{\prime}\right)^{2}|x|^{2}+\epsilon^{2}} \leq 0,
$$

since $\Psi^{\prime} \geq 0$. Moreover,

$$
|B|=\left|\Psi^{\prime \prime}\right| \frac{\epsilon^{2}|x|^{2}}{\left(\Psi^{\prime}\right)^{2}|x|^{2}+\epsilon^{2}} .
$$


Now if $\left|\Psi^{\prime}\right| \leq \epsilon$, then

$$
\begin{aligned}
|B| & \leq\left|\Psi^{\prime \prime}\right||x|^{2} \leq C\left|\Psi^{\prime \prime}\right| \quad\left(\text { since } \Psi^{\prime \prime}=0 \text { if }|x| \geq 2\right) \\
& \leq C\left(\Psi^{\prime}\right)^{1 / 2} \quad(\text { by }(4.21)) \\
& \leq C \epsilon^{1 / 2} .
\end{aligned}
$$

On the other hand if $\left|\Psi^{\prime}\right| \geq \epsilon$, we have

$$
|B| \leq\left|\Psi^{\prime \prime}\right| \frac{\epsilon^{2}}{\left(\Psi^{\prime}\right)^{2}} \leq \frac{C \epsilon^{2}}{\left|\Psi^{\prime}\right|^{3 / 2}} \leq C \epsilon^{1 / 2} .
$$

Combining (4.22)-(4.25) yields

$$
v_{t}-\left(\delta_{i j}-\frac{v_{x_{i}} v_{x_{j}}}{|D x|^{2}+\epsilon^{2}}\right) v_{x_{i} x_{j}} \leq C \epsilon^{1 / 2}
$$

and so

$$
w_{t}^{\epsilon} \leq\left(\delta_{i j}-\frac{w_{x_{i}}^{\epsilon} w_{x_{j}}^{\epsilon}}{\left|D w^{\epsilon}\right|^{2}+\epsilon^{2}}\right) w_{x_{i} x_{j}}^{\epsilon} \text { in } \mathbb{R}^{n} \times(0, \infty)
$$

for

$$
w^{\epsilon}(x, t) \equiv v(x, t)-C t \epsilon^{1 / 2} .
$$

Now

$$
w^{\epsilon}(x, 0)=\Psi\left(|x|^{2} / 2\right)=0 \text { if }|x| \geq 2
$$

and

$$
w^{\epsilon}(x, 0)=\Psi\left(|x|^{2} / 2\right) \leq-1 \quad \text { if }|x| \leq 1 .
$$

Consequently, we see from (4.19) that

$$
w^{\epsilon} \leq g \text { on } \mathbb{R}^{n} \times\{t=0\} .
$$

Applying the maximum principle to (4.2), (4.3), (4.26), and (4.27), we deduce $w^{\epsilon} \leq u^{\epsilon}$ in $\mathbb{R}^{n} \times[0, \infty)$ for each $0<\epsilon<1$. Sending $\epsilon=\epsilon_{k}$ to zero, we then have

$$
\Psi\left(|x|^{2} / 2+(n-1) t\right)=v(x, t) \leq u(x, t)
$$

for all $x \in \mathbb{R}^{n}, t \geq 0$. Thus, $u \geq 0$ if $|x|^{2} / 2+(n+1) t \geq 2$. Similarly,

$$
\begin{gathered}
\tilde{w}_{t}^{\epsilon} \geq\left(\delta_{i j}-\frac{\tilde{w}_{x_{i}}^{\epsilon} \tilde{w}_{x_{j}}^{\epsilon}}{\left|D \tilde{w}^{\epsilon}\right|^{2}+\epsilon^{2}}\right) \tilde{w}_{x_{i} x_{j}}^{\epsilon} \text { in } \mathbb{R}^{n} \times(0, \infty), \\
\tilde{w}^{\epsilon} \geq g \text { on } \mathbb{R}^{n} \times(0, \infty),
\end{gathered}
$$


for $\tilde{w}^{\epsilon} \equiv-w^{\epsilon}$. As above we consequently deduce

$$
u \leq 0 \text { if }|x|^{2} / 2+(n+1) t \geq 2 .
$$

Assertion (4.13) is proved.

4. According to the uniqueness assertion Theorem 3.2, in fact the full limit $\lim _{\epsilon \rightarrow 0} u^{\epsilon}=u$ exists. Note also from Theorem 3.3 that

$$
\|u-\tilde{u}\|_{L^{\infty}\left(R^{n} \times[0, \infty)\right)}=\|g-\tilde{g}\|_{L^{\infty}\left(R^{n}\right)}
$$

if $\tilde{u}$ is the solution built as above for a smooth initial function $\tilde{g}$ satisfying (4.1).

Suppose at last $g$ satisfies (4.1), but is only continuous. We select smooth $\left\{g^{k}\right\}_{k=1}^{\infty}$, satisfying (4.1) (for the same $S$ ) so that $g^{k} \rightarrow g$ uniformly on $\mathbb{R}^{n}$. Denote by $u^{k}$ the solution of (2.5), (2.6) constructed above with initial function $g^{k}$. Utilizing (4.31) we see that the limit $\lim _{k \rightarrow \infty} u^{k}=u$ exists uniformly on $\mathbb{R}^{n} \times[0, \infty)$. According to Theorem $2.7 u$ is a weak solution of $(2.5),(2.6)$.

\section{Definition of the generalized evolution by mean curvature}

We now make precise the definition of the motion $\left\{\Gamma_{t}\right\}_{t>0}$ for a given initial hypersurface $\Gamma_{0}$. In fact, let us assume now only that

$$
\Gamma_{0} \text { is a compact subset of } \mathbb{R}^{n} \text {. }
$$

Choose then any continuous function $g: \mathbb{R}^{n} \rightarrow \mathbb{R}$ satisfying

$$
\Gamma_{0}=\left\{x \in \mathbb{R}^{n} \mid g(x)=0\right\}
$$

and

$$
g \text { constant on } \mathbb{R}^{n} \cap\{|x| \geq S\}
$$

for some $S>0$. Utilizing Theorems 3.2 and 4.1 , we see that there is a unique weak solution of the mean curvature evolution equation

$$
\begin{gathered}
u_{t}=\left(\delta_{i j}-u_{x_{i}} u_{x_{j}} /|D u|^{2}\right) u_{x_{i} x_{j}} \text { in } \mathbb{R}^{n} \times(0, \infty), \\
u=g \text { on } \mathbb{R}^{n} \times\{t=0\}
\end{gathered}
$$

with

$$
u \text { constant on } \mathbb{R}^{n} \times[0, \infty) \cap\{|x|+t \geq R\}
$$

for some $R>0$. 
Define then the compact set

$$
\Gamma_{t} \equiv\left\{x \in \mathbb{R}^{n} \mid u(x, t)=0\right\}
$$

for each $t>0$. We call $\left\{\Gamma_{t}\right\}_{t>0}$ the generalized evolution by mean curvature of the original compact set $\Gamma_{0}$.

We must first verify that $\left\{\Gamma_{t}\right\}_{t>0}$ is well defined.

Theorem 5.1. Assume $\hat{g}: \mathbb{R}^{n} \rightarrow \mathbb{R}$ is continuous, with

$$
\Gamma_{0}=\left\{x \in \mathbb{R}^{n} \mid \hat{g}(x)=0\right\}
$$

and

$$
\hat{g} \text { constant on } \mathbb{R}^{n} \cap\{|x| \geq S\} \text {. }
$$

Suppose $\hat{u}$ is the unique weak solution of(5.4)-(5.6), with $\hat{g}$ replacing $g$. Then

$$
\Gamma_{t}=\left\{x \in \mathbb{R}^{n} \mid \hat{u}(x, t)=0\right\}
$$

for each $t>0$. Consequently our definition (5.7) does not depend upon the particular choice of initial function $g$ satisfying (5.2), (5.3).

A related assertion for the level sets of solutions to homogeneous Hamilton-Jacobi PDE may be found in Evans-Souganidis [13, §7].

Proof. 1. First, we may as well assume $g \geq 0$ on $\mathbb{R}^{n}$ and thus $u \geq 0$ in $\mathbb{R}^{n} \times(0, \infty)$. Indeed, if $g$ is negative somewhere, we can consider the PDE (5.4)-(5.6) with $|g|$ replacing $g$, the unique solution of which, owing to Theorems 2.8 and 3.2 , is $|u|$. Our definition (5.7) is unchanged if we replace $u$ by $|u|$. Similarly we may suppose $\hat{g}, \hat{u} \geq 0$. Set

$$
\widehat{\Gamma}_{t}=\left\{x \in \mathbb{R}^{n} \mid \hat{u}(x, t)=0\right\} \quad(t \geq 0) .
$$

2. For $k=1,2, \ldots$ write $E_{0}=\varnothing$ and $E_{k}=\left\{x \in \mathbb{R}^{n} \mid g(x) \geq 1 / k\right\}$, so that

$$
E_{1} \subset \cdots \subset E_{k} \subset E_{k+1} \subset \cdots, \quad \mathbb{R}^{n}-\Gamma_{0}=\bigcup_{k=1}^{\infty} E_{k} .
$$

Define

$$
a_{k} \equiv \max _{R^{n}-E_{k-1}} \hat{g}>0 \quad(k=1,2, \ldots) .
$$

Then $a_{1} \geq a_{2} \geq \cdots$ and $\lim _{k \rightarrow \infty} a_{k}=0$, according to (5.8) and (5.11). Next define the continuous function $\Psi:[0, \infty) \rightarrow[0, \infty)$ satisfying

$$
\begin{gathered}
\Psi(0)=0, \\
\Psi(1 / k)=a_{k} \quad(k=1,2, \ldots), \\
\Psi \text { linear on }[1 /(k+1), 1 / k] \quad(k=1,2, \ldots), \\
\Psi \text { constant on }[1, \infty) .
\end{gathered}
$$


3. Write $\tilde{g}=\Psi(g)$ and $\tilde{u}=\Psi(u)$. Then $\tilde{u}$ solves (5.4)-(5.6), with $\tilde{g}$ replacing $g$. Now $\tilde{g}=\hat{g}=0$ on $\Gamma_{0}$. Furthermore, if $x \in E_{k}-E_{k-1}$, then

$$
\tilde{g}(x)=\Psi(g(x)) \geq \Psi(1 / k)=a_{k} \geq \hat{g}(x) \text { by }(5.12) .
$$

Thus $\tilde{g} \geq \hat{g}$ on $\mathbb{R}^{n}$. Consequently, Theorem 3.2 asserts

$$
\tilde{u}=\Psi(u) \geq \hat{u} \geq 0 \text { on } \mathbb{R}^{n} \times[0, \infty) .
$$

Thus if $x \in \Gamma_{t}$, then $\hat{u}(x, t)=0$ and so $x \in \widehat{\Gamma}_{t}$. Hence $\Gamma_{t} \subseteq \widehat{\Gamma}_{t}$. The opposite inclusion is similarly proved, and therefore $\Gamma_{t}=\widehat{\Gamma}_{t}$ for each $t>0$. q.e.d.

In light of this theorem, we can regard the mappings $\Gamma_{0} \mapsto \Gamma_{t}(t \geq 0)$ as comprising a time-dependent evolution on the collection $\mathscr{K}$ of compact subsets of $\mathbb{R}^{n}$. Let us write

$$
\mathscr{M}(t) \Gamma_{0} \equiv \Gamma_{t} \quad(t \geq 0)
$$

explicitly to display the dependence of $\Gamma_{t}$ on $t$ and $\Gamma_{0}$. Then $\mathscr{M}(t): \mathscr{K} \rightarrow$ $\mathscr{K}$ for each $t \geq 0$, and $\mathscr{M}(0)$ is the identity operator. We will call $\{\mathscr{M}(t)\}_{t \geq 0}$ the mean-curvature semigroup on $\mathscr{K}$.

To justify this terminology, let us verify the semigroup property.

Theorem 5.2. We have

$$
\mathscr{M}(t+s)=\mathscr{M}(t) \mathscr{M}(s) \quad(t, s \geq 0) .
$$

Proof. If $t, s>0$ and $\Gamma_{0} \in \mathscr{K}$, choose any continuous function $g$ satisfying (5.2), (5.3). Let $u$ be the corresponding unique weak solution of $(5.4)-(5.6)$. Then

$$
\begin{gathered}
\mathscr{M}(t+s) \Gamma_{0}=\Gamma_{t+s}=\left\{x \in \mathbb{R}^{n} \mid u(x, t+s)=0\right\}, \\
\mathscr{M}(s) \Gamma_{0}=\Gamma_{s}=\left\{x \in \mathbb{R}^{n} \mid u(x, s)=0\right\} .
\end{gathered}
$$

To compute $\mathscr{M}(t) \Gamma_{s}$ we select any continuous function $\hat{g}$ so that

$$
\Gamma_{s}=\left\{x \in \mathbb{R}^{n} \mid \hat{g}(x)=0\right\}
$$

and $\hat{g}$ is constant outside some large ball. We then find the unique weak solution $\hat{u}$ of (5.4)-(5.6) (with $\hat{g}$ replacing $g$ ) and set

$$
\mathscr{M}(t) \Gamma_{s}=\widehat{\Gamma}_{t}=\left\{x \in \mathbb{R}^{n} \mid \hat{u}(x, t)=0\right\} .
$$

According to Theorem 5.1, this construction is independent of the particular choice of $\hat{g}$ satisfying (5.17). In particular, we may as well take $\hat{g}(x)=u(x, s) \quad\left(x \in \mathbb{R}^{n}\right)$. Owing then to the uniqueness of a weak solution to (5.4)-(5.6) we have

$$
\hat{u}(x, t)=u(x, t+s) \quad\left(x \in \mathbb{R}^{n}, t>0\right) .
$$


Consequently (5.15) and (5.18) imply

$$
\mathscr{M}(t+s) \Gamma_{0}=\mathscr{M}(t) \mathscr{M}(s) \Gamma_{0},
$$

as required. This establishes (5.14). q.e.d.

Note that we make no assertions concerning continuity of the mapping $\left(t, \Gamma_{0}\right) \mapsto \mathscr{M}(t) \Gamma_{0}$.

\section{Consistency with classical motion by mean curvature}

We must now check that our generalized evolution by mean curvature agrees with the classical motion, if and so long as the latter exists. Let us therefore suppose for this section that $\Gamma_{0}$ is a smooth hypersurface, the connected boundary of a bounded open set $U \subset \mathbb{R}^{n}$. According to Hamilton [22], Gage-Hamilton [17], and Evans-Spruck [14], there exists a time $t_{*}>0$ and a family $\left\{\Sigma_{t}\right\}_{0<t<t}$ of smooth hypersurfaces evolving from $\Sigma_{0}=\Gamma_{0}$ according to classical motion by mean curvature. In particular for each $0 \leq t<t_{*}, \Sigma_{t}$ is diffeomorphic to $\Gamma_{0}$, and is the boundary of an open set $U_{t}$ diffeomorphic to $U_{0} \equiv U$.

Theorem 6.1. We have $\Sigma_{t}=\Gamma_{t}\left(0 \leq t<t_{*}\right)$, where $\left\{\Gamma_{t}\right\}_{t \geq 0}$ is the generalized evolution by mean curvature defined in $\S 5$.

Proof. 1. Fix $0<t_{0}<t_{*}$, and define then for $0 \leq t \leq t_{0}$ the (signed) distance function

$$
d(x, t) \equiv \begin{cases}-\operatorname{dist}\left(x, \Sigma_{t}\right) & \text { if } x \in U_{t}, \\ \operatorname{dist}\left(x, \Sigma_{t}\right) & \text { if } x \in \mathbb{R}^{n} \backslash \bar{U}_{t} .\end{cases}
$$

As $\Sigma \equiv \bigcup_{0 \leq t \leq t_{0}} \Sigma_{t} \times\{t\}$ is smooth, $d$ is smooth in the regions

$$
Q^{+} \equiv\left\{(x, t) \mid 0 \leq d(x, t) \leq \delta_{0}, 0 \leq t \leq t_{0}\right\}
$$

and

$$
Q^{-} \equiv\left\{(x, t) \mid-\delta_{0} \leq d(x, t) \leq 0,0 \leq t \leq t_{0}\right\}
$$

for $\delta_{0}>0$ sufficiently small.

2. Now if $\delta_{0}>0$ is small enough, for each point $(x, t) \in Q^{+}$there exists a unique point $y \in \Sigma_{t}$ verifying $d(x, t)=|x-y|$. Consider now near $(y, t)$ the smooth unit vector field $\nu \equiv D d$ pointing from $\Sigma$ into $Q^{+}$. Then

$$
d_{t}(x, t)=(\operatorname{div} \nu)(y, t)
$$

since $\left\{\Sigma_{t}\right\}_{0 \leq t \leq t_{*}}$ is a classical evolution by mean curvature. Additionally, the eigenvalues of $D^{2} d(x, t)$ are (see, e.g., Gilbarg-Trudinger [18, p. 355])

$$
\left\{\frac{-\kappa_{1}}{1-\kappa_{1} d}, \cdots, \frac{-\kappa_{n-1}}{1-\kappa_{n-1} d}, 0\right\}
$$


$\kappa_{1}, \cdots, \kappa_{n-1}$ denoting the principal curvatures of $\Sigma_{t}$ at the point $y$, calculated with respect to the unit normal field $\nu$. Thus,

$$
\Delta d(x, t)=-\sum_{i=1}^{n-1} \frac{\kappa_{i}}{1-\kappa_{i} d} .
$$

However, $(\operatorname{div} \nu)(y, t)=-\left(\kappa_{1}+\cdots+\kappa_{n-1}\right)$, and so (6.1) and (6.3) imply

$$
d_{t}-\Delta d=\left(\sum_{i=1}^{n-1} \frac{\kappa_{i}^{2}}{1-\kappa_{i} d}\right) d \quad \text { at }(x, t) .
$$

Since the quantity $\sum_{i=1}^{n-1} \kappa_{i}^{2} /\left(1-\kappa_{i} d\right)$ is uniformly bounded and $d \geq 0$ in $Q^{+}$, we deduce from (6.4) that

$$
\underline{d} \equiv \alpha e^{-\lambda t} d
$$

satisfies

$$
\underline{d}_{t}-\Delta \underline{d} \leq 0 \text { in } Q^{+}
$$

if $\lambda>0$ is fixed large enough and $\alpha>0$ (to be selected later). Furthermore, $|D d|^{2}=|\nu|^{2}=1$ and so $d_{x_{i}} d_{x_{i} x_{j}}=0$ in $Q^{+}(1 \leq j \leq n)$. The function $\underline{d}$ satisfies the same identity, whence (6.6) implies for each $\epsilon \geq 0$ that

$$
\underline{d}_{t}-\left(\delta_{i j}-\frac{\underline{d}_{x_{i}} \underline{d}_{x_{j}}}{|D \underline{d}|^{2}+\epsilon^{2}}\right) \underline{d}_{x_{i} x_{j}} \leq 0 \text { in } Q^{+} .
$$

We see therefore that $\underline{d}$ is a smooth subsolution of the approximate mean curvature evolution PDE (4.2) in $Q^{+}$.

3. Choose any Lipschitz function $g: \mathbb{R}^{n} \rightarrow \mathbb{R}^{+}$so that $g(x)=$ $\operatorname{dist}\left(x, \Sigma_{0}\right)$ near $\Sigma_{0},\{g=0\}=\Sigma_{0}$, and $g(x)$ is a positive constant for large $|x|$. For $0<\epsilon<1$ the approximating PDE (4.2), (4.3) then has a continuous solution $u^{\epsilon}$, which is smooth in $\mathbb{R}^{n} \times(0, \infty)$. Additionally we have $u^{\epsilon} \rightarrow u$ locally uniformly, where

$$
\Gamma_{t}=\left\{x \in \mathbb{R}^{n} \mid u(x, t)=0\right\}, \quad t \geq 0 .
$$

Now $u=g=\delta_{0}>0$ on $\left\{(x, 0) \mid \operatorname{dist}\left(x, \Sigma_{0}\right)=\operatorname{dist}\left(x, \Gamma_{0}\right)=\delta_{0}\right\}$; and, as $u$ is continuous, we thus have

$$
u \geq \delta_{0} / 2>0 \text { on }\left\{(x, t) \mid d(x, t)=\delta_{0}\right\}
$$

for $0 \leq t \leq t_{0}$, provided $t_{0}>0$ is small enough. Hence (6.9) implies

$$
u^{\epsilon} \geq \delta_{0} / 4 \text { on }\left\{(x, t) \mid d(x, t)=\delta_{0}\right\}
$$


for $0 \leq t \leq t_{0}, 0<\epsilon \leq \epsilon_{0}$, if $\epsilon_{0}>0$ is sufficiently small. Consequently there exists $0<\alpha<1$ so that

$$
u^{\epsilon} \geq \underline{d} \quad \text { on }\left\{(x, t) \mid d(x, t)=\delta_{0}\right\}
$$

for $0 \leq t \leq t_{0}, 0<\epsilon<\epsilon_{0}, \underline{d}$ defined by (6.5). Since $0<\alpha<1$, we have

$$
u^{\epsilon} \geq \underline{d} \text { on }\left\{(x, 0) \mid 0 \leq d(x, 0) \leq \delta_{0}\right\} .
$$

Furthermore, $g \geq 0$ implies $u^{\epsilon} \geq 0$ and so

$$
u^{\epsilon} \geq \underline{d} \text { on }\{(x, t) \mid d(x, t)=0\} .
$$

4. Combining (6.10)-(6.12) we see that $u^{\epsilon} \geq \underline{d}$ on the parabolic boundary of $Q^{+}$. Since $\underline{d}$ solves (6.7) and $u^{\epsilon}$ solves (4.2), the maximum principle implies $u^{\epsilon} \geq \underline{d}$ in $Q^{+}$. Let $\epsilon \rightarrow 0$ to conclude

$$
u>0 \text { in the interior of } Q^{+} \text {. }
$$

A similar argument using instead $\underline{d}=-\alpha e^{-\lambda t} d$ shows

$$
u>0 \text { in the interior of } Q^{-} \text {. }
$$

Since $u>0$ in $\left(R^{n} \backslash\left\{x \mid \operatorname{dist}\left(x, \Sigma_{0}\right) \leq \delta_{0}\right\}\right) \times\left[0, t_{0}\right]$, we deduce from (6.13), (6.14), and (6.8) that

$$
\Gamma_{t} \subseteq \Sigma_{t}=\{x \mid d(x, t)=0\} \quad\left(0 \leq t \leq t_{0}\right) .
$$

5. Now define a new function $\hat{g}: \mathbb{R}^{n} \rightarrow \mathbb{R}$ so that $\hat{g}(x)=d(x, 0)$ (the signed distance function to $\Sigma_{0}$ ) near $\Sigma_{0}=\Gamma_{0},\{\hat{g}=0\}=\Sigma_{0}$, and $\hat{g}(x)$ is a positive constant for large $|x|$. Let $\hat{u}$ denote the unique weak solution of (2.5), (2.6), (4.13) for this new initial function $\hat{g}$. According to Theorem 5.1

$$
\Gamma_{t}=\left\{x \in \mathbb{R}^{n} \mid \hat{u}(x, t)=0\right\} \quad(t \geq 0) .
$$

Since $\hat{g}<0$ in $U_{0}$ we know by continuity that $\hat{u}<0$ somewhere in $U_{t}$, provided $0 \leq t \leq t_{0}$ and $t_{0}$ is small. Similarly $\hat{u}>0$ somewhere in $\mathbb{R}^{n}-\bar{U}_{t}$ for each $0 \leq t \leq t_{0}$. Fix any point $x_{0} \in \Sigma_{t}$ and draw a smooth curve $C$ in $\mathbb{R}^{n}$, intersecting $\Sigma_{t}$ precisely at $x_{0}$ and connecting a point $x_{1} \in U_{t}$, where $\hat{u}\left(x_{1}, t\right)<0$, to a point $x_{2} \in \mathbb{R}^{n}-\bar{U}_{t}$, where $\hat{u}\left(x_{2}, t\right)>0$. As $\hat{u}$ is continuous, we must have $\hat{u}(x, t)=0$ for some point $x$ on the curve $C$. However (6.15) and (6.16) say that the set $\{x \mid \hat{u}(x, t)=0\}$ lies in $\Sigma_{t}$. Thus $\hat{u}\left(x_{0}, t\right)=0$. Since $x_{0}$ denotes any point on $\Sigma_{t}$ we deduce from (6.15), (6.16) that

$$
\Gamma_{t}=\Sigma_{t} \quad \text { if } 0 \leq t \leq t_{0} .
$$


We have consequently demonstrated that the classical motion $\left\{\Sigma_{t}\right\}_{0 \leq t<t_{*}}$ and the generalized motion $\left\{\Gamma_{t}\right\}_{t \geq 0}$ agree at least on some short time interval $\left[0, t_{0}\right]$.

6. Write

$$
s \equiv \sup _{0 \leq t<t_{*}}\left\{t \mid \Gamma_{\tau}=\Sigma_{\tau} \text { for all } 0 \leq \tau \leq t\right\}
$$

and suppose $s<t_{*}$. Then $\Gamma_{t}=\Sigma_{t}$ for all $0 \leq t<s$, and so, applying the continuity of the solution $u$ to (2.5) and (2.6) for $g$ as above, we have $\Gamma_{s} \supseteq \Sigma_{s}$. On the other hand if $x \in \mathbb{R}^{n}-\Sigma_{s}$, there exists $r>0$ so that $B(x, r) \subset \mathbb{R}^{n}-\Sigma_{t}$ for all $s-\epsilon \leq t \leq s, \epsilon>0$ small enough. Using this we easily deduce $x \notin \Gamma_{s}$. Hence $\Gamma_{s}=\Sigma_{s}$. But then applying steps 1-5 we deduce $\Gamma_{t}=\Sigma_{t}$ for all $s \leq t \leq s+s_{0}<t_{*}$, if $s_{0}>0$ is small enough. This contradicts the definition of $s$, and so in fact $s=t_{*}$. q.e.d.

Observe carefully that our argument in step 5 above improving (6.15) to (6.17) depends critically upon the possibility of finding an initial function $\hat{g}$ which changes sign above. Compare this with the geometric situation in Theorem 8.1 below.

\section{Geometric properties of generalized evolution by mean curvature}

We devote this section to establishing some elementary properties of the generalized evolution by mean curvature

$$
\Gamma_{0} \mapsto \mathscr{M}(t) \Gamma_{0} \equiv \Gamma_{t} \quad(t \geq 0)
$$

for $\Gamma_{0}$ a compact subset of $\mathbb{R}^{n}$.

7.1. Localization and extinction. First of all, it is known that if $\Gamma_{0}$ is the sphere $\partial B(0, R)$, then

$$
\Gamma_{t}= \begin{cases}\partial B(0, R(t)) & \text { if } 0 \leq t<t^{*} \\ \{0\} & \text { if } t=t^{*} \\ \varnothing & \text { if } t>t^{*}\end{cases}
$$

where

$$
R(t) \equiv\left(R^{2}-2(n-1) t\right)^{1 / 2} \text { for } 0 \leq t \leq t^{*} \equiv R^{2} / 2(n-1) .
$$

This assertion follows in our approach by noting $u(x, t)=$ $\Psi\left(|x|^{2}+2(n-1) t\right)$ is a weak solution of (5.4), where $\Psi: \mathbb{R} \rightarrow \mathbb{R}$ is smooth with

$$
\left\{\begin{array}{l}
\Psi^{\prime} \geq 0, \quad \Psi<0 \quad \text { on }[0, R) \\
\Psi>0 \quad \text { on }(R, 3 R), \quad \Psi \equiv 1 \quad \text { on }[3 R, \infty) .
\end{array}\right.
$$


By making comparisons with the shrinking sphere (7.2) we derive now some elementary properties of the general motion (7.1) (cf. Brakke [5, pp. 29-30]).

Theorem 7.1. (a) If $\Gamma_{0} \subset B(0, R)$, then

$$
\Gamma_{t}=\varnothing \text { for } t>R^{2} / 2(n-1) \text {. }
$$

(b) We have

$$
\Gamma_{t} \subseteq \operatorname{conv}\left(\Gamma_{0}\right) \quad(t \geq 0),
$$

where $\operatorname{conv}\left(\Gamma_{0}\right)$ denotes the convex hull of $\Gamma_{0}$.

Proof. 1. Assume first $\Gamma_{0} \subset B(0, R-\epsilon)$ for some $\epsilon>0$. Let $g: \mathbb{R}^{n} \rightarrow$ $\mathbb{R}$ be continuous, with $\Gamma_{0}=\{g=0\}, g=1$ on $\mathbb{R}^{n} \cap\{|x| \geq 2 R\}$. Set $\hat{g}(x)=\Psi\left(|x|^{2}\right)$, with $\Psi$ satisfying (7.4) selected so that $\hat{g} \leq g$ on $\mathbb{R}^{n}$. Then

$$
\hat{u} \leq u \text { on } \mathbb{R}^{n} \times[0, \infty),
$$

for $\hat{u}(x, t)=\Psi\left(|x|^{2}+2(n-1) t\right)$ and $u$ the weak solution of (5.4)-(5.6). Thus $u>0$, and so $\Gamma_{t}=\varnothing$, if $t>\frac{1}{2} R^{2} /(n-1)$.

In the general case, replace $R$ by $R+\epsilon$ in this argument and send $\epsilon \rightarrow 0$.

2. Suppose $\Gamma_{0} \subset \mathbb{R}_{+}^{n}=\left\{x_{n}>0\right\}$. Choose $R \gg 1$ so large that $\Gamma_{0} \subset B\left(R e_{n}, R\right)$, for $e_{n}=(0,0, \cdots, 0,1)$. By the argument in step 1, we deduce $\Gamma_{t} \subset B\left(R e_{n}, R(t)\right)$ for $0 \leq t \leq \frac{1}{2} R^{2} /(n-1), R(t)$ defined as above. In particular, $\Gamma_{t} \subseteq \mathbb{R}_{+}^{n}$ for all $t \geq 0$. Replacing $\mathbb{R}_{+}^{n}$ in this argument by an open half-space containing $\Gamma_{0}$, we obtain (7.6).

7.2. Comparison of different sets moving by mean curvature.

Theorem 7.2. Let $\Gamma_{0}$ and $\widehat{\Gamma}_{0}$ be compact subsets of $\mathbb{R}^{n}$, and denote by $\left\{\Gamma_{t}\right\}_{t \geq 0}$ and $\left\{\widehat{\Gamma}_{t}\right\}_{t \geq 0}$ the corresponding generalized motions by mean curvature. Suppose also

$$
\Gamma_{0} \subseteq \widehat{\Gamma}_{0}
$$

Then

$$
\Gamma_{t} \subseteq \widehat{\Gamma}_{t} \text { for each } t>0
$$

We see therefore that if a compact set $\Gamma_{0}$ lies within another $\widehat{\Gamma}_{0}$ at time zero, then the subsequent evolution $\Gamma_{t}$ of $\Gamma_{0}$ lies within the subsequent evolution $\widehat{\Gamma}_{t}$ of $\widehat{\Gamma}_{0}$, for each $t>0$. We will see in $\S 8$ that this assertion provides us with a useful tool for studying specific examples.

Proof. Choose continuous functions $g, \hat{g}: \mathbb{R}^{n} \rightarrow[0, \infty)$ so that $\Gamma_{0}=$ $\{g=0\}$ and $\widehat{\Gamma}_{0}=\{\hat{g}=0\}$, and $g$ and $\hat{g}$ are constant on $\mathbb{R}^{n} \cap\{|x| \geq S\}$ 
for some $S>0$. Replacing $g$ by $g+\hat{g}$ if necessary, we may assume

$$
\hat{g} \leq g \text { on } \mathbb{R}^{n} \text {. }
$$

Now let $\hat{u}, u$ denote the corresponding weak solutions of (5.4)-(5.6). Then (7.9) implies $0 \leq \hat{u} \leq u$ on $\mathbb{R}^{n} \times(0, \infty)$. Thus, $x \in \Gamma_{t}$ implies $x \in \widehat{\Gamma}_{t}$, and so (7.8) is valid.

Theorem 7.3. Assume $\Gamma_{0}$ and $\widehat{\Gamma}_{0}$ are nonempty compact sets, and $\left\{\Gamma_{t}\right\}_{t \geq 0}$ and $\left\{\widehat{\Gamma}_{t}\right\}_{t \geq 0}$ are the subsequent generalized motions by mean curvature. Then

$$
\operatorname{dist}\left(\Gamma_{0}, \widehat{\Gamma}_{0}\right) \leq \operatorname{dist}\left(\Gamma_{t}, \widehat{\Gamma}_{t}\right) \quad(t \geq 0) .
$$

By definition, $\operatorname{dist}\left(\Gamma_{t}, \widehat{\Gamma}_{t}\right)=+\infty$ if $\Gamma_{t}=\varnothing, \widehat{\Gamma}_{t}=\varnothing$, or both. that

$$
\left\{\begin{array}{l}
\Gamma_{0}=\{g=0\}, \quad \widehat{\Gamma}_{0}=\{g=1\}, \\
g=2 \text { on } \mathbb{R}^{n} \cap\{|x| \geq S\} \text { for some } S, \\
\operatorname{Lip}(g)=\operatorname{dist}\left(\Gamma_{0}, \widehat{\Gamma}_{0}\right)^{-1}
\end{array}\right.
$$

Then

$$
\Gamma_{t}=\{u=0\}, \quad \widehat{\Gamma}_{t}=\{u=1\},
$$

with $u$ denoting the corresponding weak solution of (5.4)-(5.6).

2 . From the contraction property Theorem 3.3 , we see that

$$
\operatorname{Lip}(u(\cdot, t)) \leq \operatorname{Lip}(g) \quad(t \geq 0) .
$$

If $\Gamma_{t} \neq \varnothing$ and $\hat{\Gamma}_{t} \neq \varnothing$, choose points $x \in \Gamma_{t}, \hat{x} \in \hat{\Gamma}_{t}$ so that

$$
|x-\hat{x}|=\operatorname{dist}\left(\Gamma_{t}, \hat{\Gamma}_{t}\right) .
$$

Then using (7.11)-(7.13) we compute

$$
1=u(\hat{x}, t)-u(x, t) \leq \operatorname{Lip}(u)|x-\hat{x}| \leq \operatorname{dist}\left(\Gamma_{0}, \widehat{\Gamma}_{0}\right)^{-1} \operatorname{dist}\left(\Gamma_{t}, \hat{\Gamma}_{t}\right) .
$$

This proves (7.10). q.e.d.

Inequality (7.10) implies in particular that two hypersurfaces evolving under generalized motion by mean curvature do not ever move closer to each other than they were initially. In particular, $\Gamma_{t} \cap \hat{\Gamma}_{t}=\varnothing$ for all $t>0$ provided $\Gamma_{0} \cap \widehat{\Gamma}_{0}=\varnothing$. Notice that this property is essential for our approach of representing the evolving surfaces as the level sets of a continuous function. 
7.3. Positive mean curvature. Now let us assume that $\Gamma_{0}$ is a smooth connected hypersurface, the boundary of a bounded open set $U \subset \mathbb{R}^{n}$. We will suppose additionally that

$$
\operatorname{div}(\nu)<0 \text { on } \Gamma_{0},
$$

$\nu$ denoting the inner unit normal vector field to $\Gamma_{0}$ (extended smoothly to some neighborhood of $\Gamma_{0}$ ). Inequality (7.14) says that $\Gamma_{0}$ has positive mean curvature with respect to the inner unit normal field. Consequently, if $\Gamma_{0}$ evolves according to mean curvature, we see from (2.2) that initially at least the motion is directed into $U$.

We show now that in fact $\Gamma_{t}$ lies in $U$ for all $t \geq 0$, and that $\Gamma_{t}$ continues to have positive mean curvature, this last statement interpreted in an appropriate weak sense.

Expanding upon a suggestion of L. Caffarelli, our idea is to solve the mean curvature equation (5.4)-(5.6) by separating variables. Indeed we will show

$$
u(x, t) \equiv v(x)-t \quad(x \in U, t>0),
$$

where $v$ is the (unique) weak solution of the stationary problem

$$
\begin{gathered}
-\left(\delta_{i j}-\left.v_{x_{i}} v_{x_{j}}|| D v\right|^{2}\right) v_{x_{i} x_{j}}=1 \text { in } U, \\
v=0 \quad \text { on } \partial U=\Gamma_{0} .
\end{gathered}
$$

We will further prove that

$$
\Gamma_{t}=\{x \in U \mid v(x)=t\} \quad(t \geq 0),
$$

so that $\Gamma_{t} \subset U \quad(t \geq 0)$ and $\Gamma_{t}=\varnothing$ for $t>t^{*} \equiv\|v\|_{L^{\infty}(U)}$. Note also that in any open region where $v$ is smooth and $|D v| \neq 0$, we can rewrite (7.16) as

$$
-\operatorname{div}(\nu)=1 /|D v|>0 \text { for } \nu \equiv D v /|D v| .
$$

As $\nu$ is the inward pointing unit normal field along $\Gamma_{t} \equiv\{v=t\}$, we informally interpret our PDE (7.16) as implying " $\Gamma_{t}$ has positive mean curvature" for $0 \leq t<t^{*}$.

To carry out the foregoing program rigorously, let us first define $v \in$ $C(\bar{U})$ to be a weak solution to (7.16) provided that if $u-\phi$ has a local maximum (minimum) at a point $x_{0} \in U$ for each $\phi \in C^{\infty}\left(\mathbb{R}^{n}\right)$, then

$$
-\left(\delta_{i j}-\phi_{x_{i}} \phi_{x_{j}} /|D \phi|^{2}\right) \phi_{x_{i} x_{j}} \leq(\geq) 1 \text { at } x_{0} \text { if } D \phi\left(x_{0}\right) \neq 0
$$


and

$$
\left\{\begin{array}{l}
-\left(\delta_{i j}-\eta_{i} \eta_{j}\right) \epsilon_{x_{i} x_{j}} \leq(\geq) 1 \text { at } x_{0} \text { for some } \eta \in \mathbb{R}^{n} \\
\text { with }|\eta| \leq 1, \text { if } D \phi\left(x_{0}\right)=0
\end{array}\right.
$$

Theorem 7.4. There exists a unique weak solution $v$ of (7.16), (7.17). Furthermore, there are constants $A, a>0$ so that

$$
\begin{gathered}
a \operatorname{dist}\left(x, \Gamma_{0}\right) \leq v(x) \leq A \operatorname{dist}\left(x, \Gamma_{0}\right) \quad(x \in \bar{U}), \\
|D v(x)| \leq A .
\end{gathered}
$$

Proof. 1. Similarly to $\S 4$, we approximate $(7.16),(7.17)$ by the uniformly elliptic PDE

$$
\begin{gathered}
-\left(\delta_{i j}-\frac{v_{x_{i}}^{\epsilon} v_{x_{j}}^{\epsilon}}{\left|D v^{\epsilon}\right|^{2}+\epsilon^{2}}\right) v_{x_{i} x_{j}}^{\epsilon}=1 \text { in } U, \\
v^{\epsilon}=0 \text { on } \partial U=\Gamma_{0}
\end{gathered}
$$

for $0<\epsilon \leq 1$. We will construct upper and lower barriers for (7.22), (7.33) of the form

$$
w(x)=\lambda g(d(x)) \quad\left(\lambda \in \mathbb{R}, d(x)=\operatorname{dist}\left(x, \Gamma_{0}\right)\right)
$$

in a neighborhood $V \equiv\left\{0<d(x)<2 \delta_{0}\right\}$ of $\Gamma_{0}$ in which $d$ is smooth. Owing to the mean curvature condition (7.14), $d$ satisfies

$$
0<b \leq-\Delta d \leq B, \quad d_{x_{i}} d_{x_{j}} d_{x_{i} x_{j}} \equiv 0
$$

in this region. We then use (7.24) to compute

$$
\begin{aligned}
M w & \equiv\left(\delta_{i j}-\frac{w_{x_{i}} w_{x_{j}}}{|D w|^{2}+\epsilon^{2}}\right) w_{x_{i} x_{j}} \\
& =\lambda\left(g^{\prime} \Delta d+g^{\prime \prime}\right)-\frac{\lambda^{3}}{\lambda^{2} g^{\prime 2}+\epsilon^{2}} g^{\prime 2} g^{\prime \prime} \\
& =\lambda g^{\prime} \Delta d+\frac{\epsilon^{2} \lambda g^{\prime \prime}}{\lambda^{2} g^{\prime 2}+\epsilon^{2}} .
\end{aligned}
$$

Choosing $g(t)=\delta_{0}^{2}-\left(t-\delta_{0}\right)^{2}$ we find from (7.24), (7.25) that $M w \geq$ $-c \lambda \delta_{0}-2 \lambda>-1$ for $\lambda$ sufficiently small. Since $w=0$ on $\partial V, w<v^{\epsilon}$ in $V$ by the maximum principle. In particular, $v^{\epsilon}(x) \geq a d(x)(x \in U)$, where the constant $a$ is independent of $\epsilon$. To obtain the corresponding upper bound, we choose

$$
g(t)=\log \left(2 \delta_{0} /\left(2 \delta_{0}-t\right)\right) .
$$


Then $g(t)$ is convex on $\left[0,2 \delta_{0}\right)$, and satisfies

$$
g(0)=0, \quad g^{\prime} \geq 1 /\left(2 \delta_{0}\right), \quad g^{\prime \prime}=g^{\prime 2}, \quad g^{\prime}\left(2 \delta_{0}\right)=+\infty .
$$

Again using (7.24)-(7.26), we find

$$
M w \leq-c \lambda+\epsilon^{2} / \lambda<-1
$$

for $\lambda$ sufficiently large. Since $\partial w / \partial v=+\infty$ on $\left\{d=2 \delta_{0}\right\}$, where $\nu$ denotes the exterior normal to $V$, we find that $v^{\epsilon}<w$ in $V$ by a simple variant of the maximum principle. This gives the estimate

$$
v^{\epsilon}(x) \leq A d(x) \quad(x \in V) .
$$

To complete our preliminary estimates, we observe that (7.27) implies $\left|D v^{\epsilon}\right| \leq A$ on $\Gamma_{0}$. By differentiating (7.22) with respect to $x_{l}$, we see that any derivative $v_{x_{l}}^{\epsilon}$ achieves its maximum and minimum on $\Gamma_{0}$. Thus $\left|D v^{\epsilon}\right|$ is uniformly bounded in $U$ and in particular $v^{\epsilon} \leq A d$ in $U$.

2. As a consequence of step 1, we derived the uniform bounds

$$
\sup _{0<\epsilon \leq 1}\left\|v^{\epsilon}\right\|_{C^{0,1}(U)}<\infty
$$

Hence we may extract a subsequence $\left\{v^{\epsilon_{k}}\right\}_{k=1}^{\infty} \subset\left\{v^{\epsilon}\right\}_{0<\epsilon \leq 1}$ so that $\epsilon_{k} \rightarrow 0$ and $v^{\epsilon_{k}} \rightarrow v$ uniformly on $\bar{U}$. As in the proof of Theorem 4.2, we verify that $v$ is a weak solution of (7.16).

3 . The uniqueness of this weak solution $v$ will follow from the characterization of $\left\{\Gamma_{t}\right\}_{t \geq 0}$ below.

Theorem 7.5. Let $\left\{\Gamma_{t}\right\}_{t \geq 0}$ denote the generalized evolution by mean curvature starting with $\Gamma_{0}$. Then $\Gamma_{t}=\{x \in U \mid v(x)=t\}$ for each $t \geq 0$.

Proof. 1. Define $u(x, t) \equiv v(x)-t$ for $x \in U, t>0$. It is then straightforward to verify that $u$ is a weak solution of the mean curvature evolution equation

$$
u_{t}=\left(\delta_{i j}-u_{x_{i}} u_{x_{j}} /|D u|^{2}\right) u_{x_{i} x_{j}} \text { in } U \times(0, \infty) .
$$

Set

$$
\widehat{\Gamma}_{t} \equiv\{x \in U \mid v(x)=t\}=\{x \in U \mid u(x, t)=0\} \quad(t>0) .
$$

2. Now let

$$
\hat{u}(x, t) \equiv|u(x, t)|=|v(x)-t| \quad(x \in U, t>0) .
$$

In view of Theorem $2.8, \hat{u}$ is a weak solution of

$$
\left\{\begin{array}{l}
\hat{u}_{t}=\left(\delta_{i j}-\hat{u}_{x_{i}} \hat{u}_{x_{j}} /|D \hat{u}|^{2}\right) \hat{u}_{x_{i} x_{j}} \text { in } U \times(0, \infty), \\
\hat{u}=t \text { on } \partial U \times[0, \infty), \\
\hat{\mu}=v \text { on } \bar{U} \times\{t=0\} .
\end{array}\right.
$$


3. Choose any smooth function $g: \mathbb{R}^{n} \rightarrow \mathbb{R}$ so that

$$
\left\{\begin{array}{l}
\Gamma_{0}=\{g=0\}, \quad g \geq 0, \quad D g \neq 0 \text { on } \Gamma_{0}, \\
g \text { is constant on } \mathbb{R}^{n} \cap\{|x| \geq S\} \text { for some } S>0 .
\end{array}\right.
$$

Let $w \geq 0$ be the unique weak solution of

$$
\left\{\begin{array}{l}
w_{t}=\left(\delta_{i j}-w_{x_{i}} w_{x_{j}} /|D w|^{2}\right) w_{x_{i} x_{j}} \text { in } \mathbb{R}^{n} \times(0, \infty), \\
w=g \text { on } \mathbb{R}^{n} \times\{t=0\}
\end{array}\right.
$$

so that

$$
\Gamma_{t}=\left\{x \in \mathbb{R}^{n} \mid w(x, t)=0\right\} \quad(t \geq 0) .
$$

According to our construction in $\S 4, w$ is Lipschitz in $t$, and thus

$$
|w(x, t)| \leq C t \quad\left(x \in \Gamma_{0}, t>0\right)
$$

for some constant $C$.

4. Employing now (7.21), we see that $\underline{w} \equiv \alpha w$ satisfies

$$
\underline{w} \leq \begin{cases}v & \text { on } \bar{u} \times\{t=0\} \\ t & \text { on } \partial U \times(0, \infty)\end{cases}
$$

if $\alpha>0$ is sufficiently small.

Now the proof of our Comparison Theorem 3.2 can be modified to show from (7.30), (7.32) that $0 \leq \underline{w} \leq \hat{u}$ in $U \times[0, \infty)$. Thus $x \in \widehat{\Gamma}_{t}$ implies $x \in \Gamma_{t}$, and so $\widehat{\Gamma}_{t} \subseteq \Gamma_{t}$ for $t \geq 0$. Similarly, let us set

$$
\bar{w} \equiv \beta w
$$

for some large constant $\beta$. Now (7.14) yields that if $t_{0}$ is sufficiently small, then

$$
\Gamma_{t} \subset U \quad\left(0 \leq t \leq t_{0}\right)
$$

Since $\partial U=\Gamma_{0}$, we may employ (7.35) and the semigroup property (5.14) to conclude $\Gamma_{t} \subset U(t>0)$. In particular, $w>0$ on $\partial U \times(0, \infty)$. Consequently, for any $T>0$ we may choose $\beta$ so large that $\bar{w}$ defined by (7.34) satisfies $\hat{u} \leq \bar{w}$ on $U \times[0, T]$. Hence as above we find

$$
\Gamma_{t}=\widehat{\Gamma}_{t} \quad(0 \leq t \leq T) .
$$

7.4. Convexity. We next recover certain assertions of Huisken [23], by suitably adapting various methods of Korevaar [29] and Kennington [27] for studying the convexity of solutions to nonlinear elliptic PDE. Kennington had previously proposed this method in [28] (see also the concluding remarks in Trudinger [36]). 
Theorem 7.6. Assume $\Gamma_{0}$ is the boundary of a smooth convex bounded open set $U$. Then there exists a time $t^{*}>0$ such that $\Gamma_{t}$ is the boundary of a convex, nonempty open set for $0 \leq t<t^{*}$ and $\Gamma_{t}$ is empty for $t>t^{*}$.

Proof. 1. Because of $\S 7.3$ it suffices to consider the stationary PDE

$$
\left\{\begin{array}{l}
-\left(\delta_{i j}-v_{x_{i}} v_{x_{j}} /|D v|^{2}\right) v_{x_{i} x_{j}}=1 \text { in } U, \\
v=0 \text { on } \Gamma_{0}=\partial U .
\end{array}\right.
$$

We will show that $\{x \in U \mid v(x)>t\}$ is convex for $0 \leq t<t^{*}, t^{*}=$ $\|v\|_{L^{\infty}}$. In fact, we will show that $\sqrt{v}$ is concave.

Formally, if $w=\sqrt{v}$ and $v$ satisfies (7.36), then $w$ solves

$$
-\left(\delta_{i j}-w_{x_{i}} w_{x_{j}} /|D w|^{2}\right) w_{x_{i} x_{j}}=1 / 2 w \text { in } U \text {. }
$$

This suggests we consider approximations $w^{\epsilon}=\sqrt{v^{\epsilon}}$ satisfying

$$
\begin{gathered}
M w^{\epsilon} \equiv\left(\delta_{i j}-\frac{w_{x_{i}}^{\epsilon} w_{x_{j}}^{\epsilon}}{\left|D w^{\epsilon}\right|^{2}+\epsilon^{2}}\right) w_{x_{i} x_{j}}^{\epsilon}=-\frac{1}{2 w^{\epsilon}} \quad \text { in } U, \\
w^{\epsilon}=0 \quad \text { on } \Gamma_{0}, \\
-\left(\delta_{i j}-\frac{v_{x_{i}}^{\epsilon} v_{x_{j}}^{\epsilon}}{\left|D v^{\epsilon}\right|^{2}+4 \epsilon^{2} v^{\epsilon}}\right) v_{x_{i} x_{j}}^{\epsilon}=\frac{-2 \epsilon^{2}\left|D v^{\epsilon}\right|^{2}}{\left|D v^{\epsilon}\right|^{2}+4 \epsilon^{2} v^{\epsilon}}+1 \quad \text { in } U .
\end{gathered}
$$

Because the convexity arguments are very sensitive to the form of the equation, we are forced into making a nice approximation $w^{\epsilon}$ to (7.37) and then making due with nastier approximations $v^{\epsilon}$ to (7.36).

2. We first demonstrate the existence of a solution $w^{\epsilon} \in C^{2}(U) \cap$ $C^{1 / 2}(\bar{U})$ to (7.38). Consider therefore the PDE

$$
\begin{aligned}
M s^{\epsilon, \delta} & \equiv\left(\delta_{i j}-\frac{w_{x_{i}}^{\epsilon, \delta} w_{x_{j}}^{\epsilon, \delta}}{\left|D w^{\epsilon, \delta}\right|^{2}+\epsilon^{2}}\right) w_{x_{i} x_{j}}^{\epsilon, \delta}=-\frac{1}{2\left(w^{\epsilon, \delta}+\delta\right)} \text { in } U, \\
w^{\epsilon, \delta} & =0 \text { on } \Gamma_{0},
\end{aligned}
$$

which has a unique smooth solution $w^{\epsilon, \delta} \geq 0$.

Choose a large ball $B(p, R)$ containing $U$ with $\operatorname{dist}(p, U) \geq R / 2$, and let $r \equiv|x-p|$. Set $w \equiv(2 R-r)$. Then

$$
M w+\frac{1}{2(w+\delta)}=-\frac{(n-1)}{r}+\frac{1}{2(2 R-r)} \leq-\frac{(n-1)}{R}+\frac{1}{3 R}<0 .
$$

Since $w>0$ on $\partial U, w>w^{\epsilon, \delta}$ in $U$ by the maximum principle. Hence

$$
0 \leq w^{\epsilon, \delta}<2 R \text { in } U
$$

with $R$ independent of $\epsilon$ and $\delta$. 
Next, let $w \equiv \lambda \sqrt{d}$ in $V=\left\{0<d(x)<\delta_{0}\right\}$. Using formula (7.25) of $\S 7.3($ with $g(t)=\sqrt{t}$ ) we find

$$
M w+\frac{1}{2(w+\delta)} \leq-\frac{c \lambda}{\sqrt{d}}+\frac{1}{2(\lambda \sqrt{d}+\delta)}<0
$$

for $\lambda$ sufficiently large. If in addition, we choose $\lambda$ so that $\lambda \sqrt{\delta_{0}} \geq 2 R$, then $w \geq w^{\epsilon, \delta}$ on $\partial V$, and thus $w \geq w^{\epsilon, \delta}$ on $V$ by the maximum principle. In particular

$$
0 \leq w^{\epsilon, \delta} \leq A \sqrt{d} \text { in } U,
$$

with $A$ independent of $\epsilon$ and $\delta$.

Estimate (7.42) implies that

$$
\left|w^{\epsilon, \delta}(x)-w^{\epsilon, \delta}(y)\right| \leq C|x-y|^{1 / 2} \text { if } x \in U, y \in \Gamma_{0},
$$

with $C$ independent of $\epsilon$ and $\delta$. We show that (7.43) holds for all $x, y \in U$ by the following well-known argument. Given $x, y \in U$ we set $\tau \equiv y-x, U_{\tau} \equiv\left\{z \in \mathbb{R}^{n} \mid z-\tau \in U\right\}$, and $w_{\tau}^{\epsilon, \delta}(z) \equiv w^{\epsilon, \delta}(z-\tau)$. Note that $U_{\tau}$ is open and nonempty since $y \in U_{\tau}$. On $U \cap U_{\tau}$, both $w^{\epsilon, \delta}$ and $w_{\tau}^{\epsilon, \delta}$ satisfy (7.40) and hence the difference $w=w^{\epsilon, \delta}-w_{\tau}^{\epsilon, \delta}$ satisfies a linear elliptic equation of the form $L w+c(x) w=0$ with $c(x) \geq 0$. Hence by the maximum principle,

$$
|w(y)| \leq \max _{z \in \partial\left(U \cap U_{\tau}\right)}|w(z)| \quad\left(y \in U \cap U_{\tau}\right) .
$$

Since for $z \in \partial\left(U \cap U_{\tau}\right)$ either $z \in \partial U$ or $z-\tau \in \partial U$, we have by (7.43) that

$$
\left|w^{\epsilon, \delta}(y)-w^{\epsilon, \delta}(x)\right|=\left|w^{\epsilon, \delta}(y)-w_{\tau}^{\epsilon, \delta}(y)\right| \leq C|x-y|^{1 / 2} .
$$

Finally, in order to pass to the limit for a sequence $\delta_{k} \searrow 0$, we need to establish some interior estimates for $w_{k}=w^{\epsilon, \delta_{k}}$. Let $W \subset \subset U$. Then we claim

$$
\left\|w_{k}\right\|_{C^{2+\alpha}(W)} \leq M\left(\epsilon, \operatorname{dist}\left(W, \Gamma_{0}\right)\right)
$$

with $M$ independent of $\delta_{k}$. By Schauder theory, (7.45) follows from an interior gradient estimate

$$
\left\|D w_{k}\right\|_{L^{\infty}(W)} \leq C\left(\epsilon, \operatorname{dist}\left(W, \Gamma_{0}\right)\right),
$$

which in turn follows from Gilbarg-Trudinger [18, Theorem 15.5]. Therefore, we have established the existence of a (unique) solution $w^{\epsilon}$ of (7.38), 
and in addition the estimates

$$
\begin{gathered}
0 \leq w^{\epsilon} \leq A \sqrt{d}, \quad 0 \leq w^{\epsilon} \leq 2 R, \\
\left|w^{\epsilon}(x)-w^{\epsilon}(y)\right| \leq C|x-y|^{1 / 2},
\end{gathered}
$$

with $A, C, R$ independent of $\epsilon$.

3. Before we proceed to the proof of the concavity of $w^{\epsilon}$, we shall need to establish the lower bound

$$
w^{\epsilon} \geq a d
$$

with $a$ independent of $\epsilon$.

Consider $w \equiv \lambda g(d)$ in $V=\left\{0<d(x)<2 \delta_{0}\right\}$ with $g(t)=$ $\left(\delta_{0}^{2}-\left(t-\delta_{0}\right)^{2}\right)^{1 / 2}$. Then from formulas (7.24) and (7.25) we find

$$
\begin{aligned}
M w & \geq-\frac{\lambda \delta_{0}}{g}\left(c+\frac{\epsilon^{2} \delta_{0}}{\lambda^{2}\left(d-\delta_{0}\right)^{2}+\epsilon^{2}\left(\delta_{0}^{2}-\left(d-\delta_{0}\right)^{2}\right)}\right) \\
& \geq-\frac{\lambda \delta_{0}}{g}\left(c+\frac{1}{\delta_{0}}\right) \quad \text { for } \lambda \geq \epsilon,
\end{aligned}
$$

and so

$$
M w+\frac{1}{2 w} \geq-\frac{\lambda}{g}\left(\delta_{0} c+1\right)+\frac{1}{2 \lambda g} \geq 0
$$

for $\epsilon^{2} \leq \lambda^{2}=2\left(\delta_{0} c+1\right)$. With this choice, we see $w^{\epsilon} \geq w$ in $\{0<$ $\left.d(x)<2 \delta_{0}\right\}$, and as in $\S 7.3$ the estimate (7.47) follows easily.

4. We can now show that $w^{\epsilon}$ is concave. For $x, y \in \bar{U}$ set $z=$ $\lambda x+(1-\lambda) y, \lambda \in(0,1)$ being fixed. The concavity function of $w^{\epsilon}$ is defined by

$$
\mathscr{C}^{\lambda}(x, y) \equiv w^{\epsilon}(z)-\lambda w^{\epsilon}(x)-(1-\lambda) w^{\epsilon}(y) \quad(x, y \in U) .
$$

The fundamental concavity maximum principle for $\mathscr{C}$ was established by Korevaar [29] for a large class of elliptic equations. The case at hand fails to satisfy Korevaar's condition. However, Kennington's improved concavity maximum principle [27, Theorem 3.1$]$ does apply and so the infimum of $\mathscr{C}^{\lambda}$ is not attained on $U \times U$.

To complete the proof we must essentially show that $w^{\epsilon}$ is concave near $\Gamma_{0}$. Since $w^{\epsilon}=\sqrt{v^{\epsilon}}$ satisfies (7.38), (7.39), it is straightforward to see that $v^{\epsilon} \in C^{2+\alpha}(\bar{U})$ and $D v^{\epsilon} \cdot \nu \geq a>0$ for $\nu$ the interior normal to $\Gamma_{0}$. Using the strict convexity of $U$ it is easy to check that

$$
w_{l l}^{\epsilon}=\frac{1}{2 \sqrt{v^{\epsilon}}} v_{l l}^{\epsilon}-\frac{\left(v_{l l}^{\epsilon}\right)^{2}}{4\left(v^{\epsilon}\right)^{3 / 2}}
$$


is strictly negative near $\Gamma_{0}$. It follows easily that $\mathscr{C}^{\lambda} \geq 0$ on $U \times U$ (for complete details, see Korevaar [29, Lemma 2.4] or Caffarelli-Spruck [6, Theorem 3.1]). This completes the proof that $w^{\epsilon}$ is concave.

5. Since $w^{\epsilon}$ is concave, it follows that $\left|D w^{\epsilon}\right| \neq 0$ on each level set of $w^{\epsilon}$ below the maximum of $w^{\epsilon}$. Hence all these level sets are smooth convex hypersurfaces.

We claim that these level sets have uniformly bounded principal curvatures. To see this, it suffices because of the convexity of these level sets to know that the mean curvature $\mathscr{H}$ with respect to the inward normal is uniformly bounded. But

$$
\begin{aligned}
\mathscr{H}\left|D w^{\epsilon}\right| & =-\left(\delta_{i j}-\frac{w_{x_{i}}^{\epsilon} w_{x_{j}}^{\epsilon}}{\left|D w^{\epsilon}\right|^{2}}\right) w_{x_{i} x_{j}}^{\epsilon} \\
& =\frac{1}{2 w^{\epsilon}}+w_{x_{i}}^{\epsilon} w_{x_{j}}^{\epsilon} w_{x_{i} x_{j}}^{\epsilon}\left(\frac{1}{\left|D w^{\epsilon}\right|^{2}}-\frac{1}{\left|D w^{\epsilon}\right|^{2}+\epsilon^{2}}\right) .
\end{aligned}
$$

Since $w^{\epsilon}$ is concave we conclude that $0 \leq \mathscr{H} \leq 1 / 2 w^{\epsilon}\left|D w^{\epsilon}\right|$, and therefore $\mathscr{H}$ is uniformly bounded on each of the level sets below the maximum of $w^{\epsilon}$.

6. We complete the proof of Theorem 7.6 by showing that $v^{\epsilon} \rightarrow v$ uniformly on $\bar{U}$, where $v$ is the unique solution of (7.36) constructed in Theorem 7.4.

Since $w^{\epsilon}$ satisfies (7.38), $v^{\epsilon}$ satisfies

$$
\left|v^{\epsilon}(x)-v^{\epsilon}(y)\right| \leq 4 R C|x-y|^{1 / 2}, \quad x, y \in U .
$$

Hence, we may choose a sequence $\epsilon_{k} \rightarrow 0$ with $v^{\epsilon_{k}} \rightarrow v$ uniformly on $\bar{U}$. We assert that $v$ is a weak solution of (7.36). As before, it suffices to consider $\phi \in C^{\infty}\left(\mathbb{R}^{n}\right)$ with $v-\phi$ having a strict local maximum at a point $x_{0} \in U$. As $v^{\epsilon_{k}} \rightarrow v$ uniformly near $x_{0}, v^{\epsilon_{k}}-\phi$ has a local maximum at a point $x_{k}$, with $x_{k} \rightarrow x_{0}$ as $k \rightarrow \infty$.

Since $v^{\epsilon_{k}}$ and $\phi$ are smooth, we have

$$
D v^{\epsilon_{k}}=D \phi, \quad D^{2} v^{\epsilon_{k}} \leq D^{2} \phi \quad \text { at } x_{k} .
$$

Thus (7.39) implies

$$
-\left(\delta_{i j}-\frac{\phi_{x_{i}} \phi_{x_{j}}}{|D \phi|^{2}+4 \epsilon_{k}^{2} v^{\epsilon_{k}}}\right) \phi_{x_{i} x_{j}} \leq-2 \epsilon_{k}^{2} \frac{|D \phi|^{2}}{|D \phi|^{2}+4 \epsilon_{k}^{2} v^{\epsilon_{k}}}+1
$$

at $x_{k}$. Suppose first $D \phi\left(x_{0}\right) \neq 0$. Then $D \phi\left(x_{k}\right) \neq 0$ for large $k$. Consequently we may pass to the limit in (7.48) (since $0 \leq v^{\epsilon_{k}} \leq 4 R^{2}$ ) to 
deduce

$$
-\left(\delta_{i j}-\frac{\phi_{x_{i}} \phi_{x_{j}}}{|D \phi|^{2}}\right) \phi_{x_{i} x_{j}} \leq 1 \text { at } x_{0} \text {. }
$$

Next, assume instead $D \phi\left(x_{0}\right)=0$ and set

$$
\eta^{k} \equiv \frac{D \phi\left(x_{k}\right)}{\left(\left|D \phi\left(x_{k}\right)\right|^{2}+4 \epsilon_{k}^{2} v^{\epsilon_{k}}\right)^{1 / 2}}
$$

so that $(7.48)$ becomes

$$
-\left(\delta_{i j}-\eta_{i}^{k} \eta_{j}^{k}\right) \phi_{x_{i} x_{j}} \leq-2 \epsilon_{k}^{2} \frac{|D \phi|^{2}}{|D \phi|^{2}+4 \epsilon_{k}^{2} v_{k}^{\epsilon}}+1 \text { at } x_{k} .
$$

Since $\left|\eta^{k}\right| \leq 1$, we may pass to a subsequence and reindex if necessary to ensure $\eta_{k} \rightarrow \eta$ in $\mathbb{R}^{n}$ for some $|\eta| \leq 1$. Sending $k$ to infinity in (7.49) we discover

$$
-\left(\delta_{i j}-\eta_{i} \eta_{j}\right) \phi_{x_{i} x_{j}} \leq 1 \text { at } x_{0} .
$$

Consequently $v$ is a weak subsolution. Similarly, we find that $v$ is a weak supersolution, and the proof of Theorem 7.6 is complete.

Remark 7.7. We have shown that if $\Gamma_{0}$ is smooth, then $\Gamma_{t}$ is a $C^{1,1}$ convex hypersurface. In a subsequent paper, we will demonstrate that for arbitrary convex $\Gamma_{0}$, the surfaces $\left\{\Gamma_{t}\right\}_{t \geq 0}$ are actually smooth. Once this smoothness is demonstrated, it follows from the work of Huisken [23] that the $\left\{\Gamma_{t}\right\}_{t \geq 0}$ are strictly convex and shrink to a point.

\section{Examples, pathologies, and conjectures}

In this concluding section, we note various odd behavior allowed by our generalized mean curvature flow

$$
\Gamma_{0} \mapsto \mathscr{M}(t) \Gamma_{0}=\Gamma_{t} \quad(t \geq 0),
$$

and set forth some related conjectures.

8.1. Instantaneous extinction. Suppose $\Sigma_{0}$ is the smooth, connected boundary of a bounded open subset $U \subset \mathbb{R}^{n}$, and let $\Gamma_{0}$ be a compact subset of $\Sigma_{0}$. If $\Gamma_{0}=\Sigma_{0}$, then we know from Theorem 6.1 that, at least for small times $t>0, \Gamma_{t}$ is the classical evolution via mean curvature.

What happens if $\Gamma_{0}$ is a proper subset of $\Sigma_{0}$ ?

Theorem 8.1. Assume that $\Gamma_{0}$ is compact, $\Gamma_{0} \subseteq \Sigma_{0}, \Gamma_{0} \neq \Sigma_{0}$. Then

$$
\Gamma_{t}=\varnothing \text { for each } t>0 \text {. }
$$


If we take $\Gamma_{0}$ to be, say, $\Sigma_{0}$ with a small disk $D$ removed, we may informally regard (8.1) as asserting $\Gamma_{0}$ "pops" instantly. In this heuristic interpretation, we may think of $\Gamma_{0}$ as somehow having so much mean curvature concentrated along its boundary within $\Sigma_{0}$ that the hole then widens infinitely fast (see Figure 5).

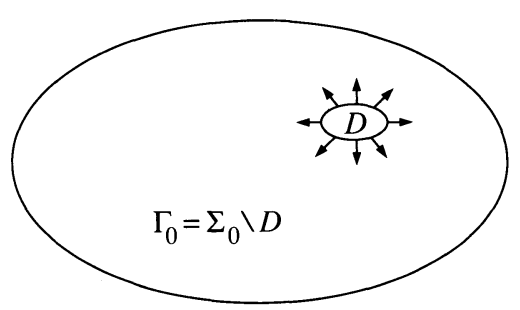

FIGURE 5

The proof of Theorem 8.1 will be given after the next assertion, of independent interest. Assume now that $\hat{\Sigma}_{0}$ is the smooth connected boundary of a bounded open set $\widehat{U} \subset \mathbb{R}^{n}$ and that

$$
\widehat{\Sigma}_{0} \subset \bar{U},
$$

with $\Sigma_{0}$ and $U$ as above. Thus the surface $\widehat{\Sigma}_{0}$ lies within the closed region $\bar{U}$ enveloped by $\Sigma_{0}$. Suppose further that

$$
\widehat{\Sigma}_{0} \neq \Sigma_{0} \text {. }
$$

Then choose a time $t_{0}>0$ so small that the classical evolutions $\left\{\Sigma_{t}\right\}$ and $\left\{\widehat{\Sigma}_{t}\right\}$ starting at $\Sigma_{0}$ and $\widehat{\Sigma}_{0}$, respectively, exist at least for times $0 \leq t \leq t_{0}$.

Theorem 8.2. We have

$$
\Sigma_{t} \cap \widehat{\Sigma}_{t}=\varnothing \text { for } 0<t \leq t_{0} .
$$

We are thus asserting that even if $\Sigma_{0}$ and $\widehat{\Sigma}_{0}$ coincide except for a very small region (see Figure 6), then for any positive $t>0$ the subsequent evolutions will have completely broken apart (as in Figure 7). The point

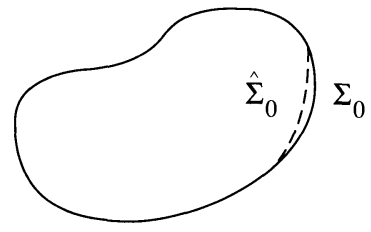

FIGURE 6

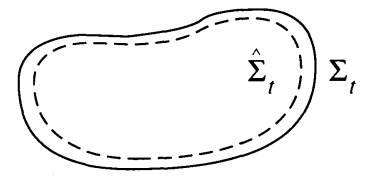

FIGURE 7 
is that the PDE describing evolution by mean curvature is "uniformly parabolic along the surface" and thus admits infinite propagation speed for disturbances.

We will give the proof of Theorem 8.2 (as well as a new proof of the short time existence of classical mean curvature flow) in a separate paper [14]. Another proof follows by covering $\Sigma_{0}$ and $\widehat{\Sigma}_{0}$ by overlapping balls small enough so that the restrictions of $\Sigma_{t}$ and $\widehat{\Sigma}_{t}$ to each ball can be written as graphs. Since the equation for the height function is uniformly parabolic for small $t_{0}$, and since $\widehat{\Sigma}_{0} \neq \Sigma_{0}$, in at least one of the balls the surfaces $\Sigma_{t}$ and $\widehat{\Sigma}_{t}$ must instantly separate. Thus in each ball the surfaces must also separate.

Proof of Theorem 8.1. Given $\Gamma_{0}$ and $\Sigma_{0}$ as in Theorem 8.1 we may choose a smooth, nearby surface $\widehat{\Sigma}_{0}$ to $\Sigma_{0}$ satisfying (8.2), (8.3), and $\Gamma_{0} \subset \widehat{\Sigma}_{0}$. Then owing to Theorem 7.2 we have $\Gamma_{t} \subseteq \Sigma_{t} \cap \widehat{\Sigma}_{t}$ for small $t>0$. Assertion (8.1) now follows from (8.4).

8.2. Development of an interior. The foregoing demonstrates that a "large" initial set $\Gamma_{0}$ can instantly vanish under the generalized mean curvature flow. An opposite and perhaps more surprising phenomenon is that the set $\Gamma_{t}$ for $t>0$ may develop an interior, even if $\Gamma_{0}$ had none.

The simplest example occurs if we take $\Gamma_{0}$ to be the union of the coordinate axes in the plane $\mathbb{R}^{2}$ (Figure 8). (Ignore for the moment that $\Gamma_{0}$ is not compact and so our theory in $\S 5$ is not really applicable.) To discover, heuristically at least, the subsequent evolution of $\Gamma_{0}$, consider instead the simpler figure as drawn in Figure 9. As for instance in Brakke [5, Figure 3] we expect this corner to evolve to the shape depicted in Figure 10 for times $t>0$. Since $\Gamma_{0}$ is composed of four rotated copies of this corner, we expect from Theorem 7.2 that $\Gamma_{t}$ will look like the shape in Figure 11. This assertion is at variance with Brakke [5, Figure 5]. Our $\Gamma_{t}$ presumably contains the set shown in Figure 12, which he draws as one of the (nonunique!) evolutions for $\Gamma_{0}$. We conjecture that our $\Gamma_{t}$ contains all of the evolutions of $\Gamma_{0}$ allowed for by Brakke.

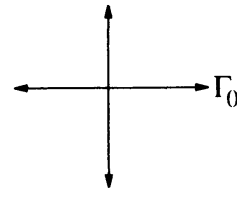

FIGURE 8

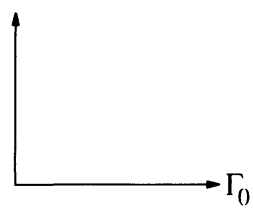

FIGURE 9

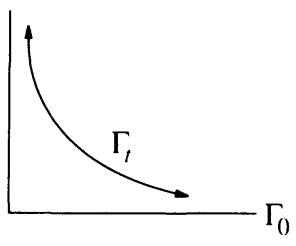

FigURE 10 


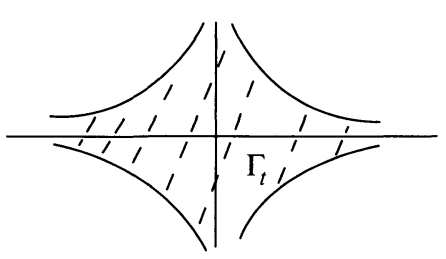

FIGURE 11

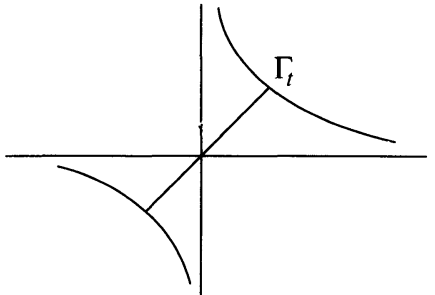

FIGURE 12

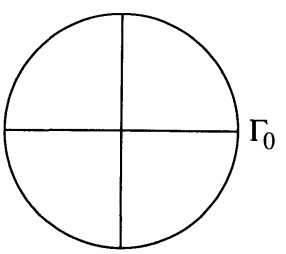

FIGURE 13

The discussion above can be modified to apply to various compact figures $\Gamma_{0}$, to which our theory does apply. We leave it to the reader to provide at least a heuristic proof that the set $\Gamma_{0} \subset \mathbb{R}^{2}$ as drawn in Figure 13 will develop an interior.

Observe by the way that our approach regards a "figure eight" in $R^{2}$ as being embedded with a singularity at the crossing point. We in particular do not interpret this shape as an immersed circle, and consequently model its evolution completely differently than [1], [3], [11], etc.

We conjecture that if $\Gamma_{0}=\Sigma_{0}$ is, as above, the boundary of a smooth open set, then $\Gamma_{t}$ will never have an interior.

\section{References}

[1] U. Abresch \& J. Langer, The normalized curve shortening flow and homothetic solutions, J. Differential Geometry 23 (1986) 175-196.

[2] W. Allard, On the first variation of a varifold, Ann. of Math. (2) 95 (1972) 417-491.

[3] S. Angenent, Parabolic equations for curves on surfaces. I, II, preprint.

[4] E. Bombieri, E. DeGiorgi \& E. Giusti, Minimal cones and the Bernstein problem, Invent. Math. 7 (1969) 243-268.

[5] K. A. Brakke, The motion of a surface by its mean curvature, Princeton Univ. Press, Princeton, NJ, 1978.

[6] L. A. Caffarelli \& J. Spruck, Convexity properties of solutions to some classical variational problems, Comm. Partial Differential Equations 7 (1982) 1337-1379.

[7] Y.-G. Chen, Y. Giga \& S. Goto, Uniqueness and existence of viscosity solutions of generalized mean curvature flow equations, preprint, 1989.

[8] M. G. Crandall, L. C. Evans \& P.-L. Lions, Some properties of viscosity solutions of Hamilton-Jacobi equations, Trans. Amer. Math. Soc. 282 (1984) 487-502. 
[9] M. G. Crandall \& P.-L. Lions, Viscosity solutions of Hamilton-Jacobi equations, Trans. Amer. Math. Soc. 277 (1983) 1-42.

[10] K. Ecker \& G. Huisken, Mean curvature evolution of entire graphs, preprint, 1988.

[11] C. L. Epstein \& M. I. Weinstein, A stable manifold theorem for the curve shortening equation, Comm. Pure Appl. Math. 40 (1987) 119-139.

[12] L. C. Evans, A convergence theorem for solutions of nonlinear second order elliptic equations, Indiana Univ. Math. J. 27 (1978) 875-887.

[13] L. C. Evans \& P. E. Souganidis, Differential games and representation formulas for solutions of Hamilton-Jacobi-Isaacs equations, Indiana Univ. Math. J. 33 (1984) 773-797.

[14] L. C. Evans \& J. Spruck, Motion of level sets by mean curvature. II, Trans. Amer. Math. Soc. (to appear).

[15] M. Gage, An isoperimetric inequality with applications to curve shortening, Duke Math. J. 50 (1983) 1225-1229.

[16] _ Curve shortening makes convex curves circular, Invent. Math. 76 (1984) 357-364.

[17] M. Gage \& R. S. Hamilton, The heat equation shrinking convex plane curves, J. Differential Geometry 23 (1986) 69-96.

[18] D. Gilbarg \& N. S. Trudinger, Elliptic partial differential equations of second order, Springer, Berlin, 1983.

[19] M. Grayson, The heat equation shrinks embedded plane curves to round points, J. Differential Geometry 26 (1987) 285-314.

[20] _, A short note on the evolution of surfaces via mean curvature, Duke Math. J. 58 (1989) 555-558.

[21] _ , The shape of a figure-eight under the curve shortening flow, Invent. Math. 96 (1989) 177-180.

[22] R. S. Hamilton, Three manifolds with positive Ricci curvature, J. Differential Geometry 17 (1982) 255-306.

[23] G. Huisken, Flow by mean curvature of convex surfaces into spheres, J. Differential Geometry 20 (1984) 237-266.

[24] H. Ishii, On uniqueness and existence of viscosity solutions of fully nonlinear second order elliptic PDE's, Comm. Pure Appl. Math. 42 (1989) 15-45.

[25] R. Jensen, The maximum principle for viscosity solutions of fully nonlinear second order partial differential equations, Arch. Rational Mech. Anal. 101 (1988) 1-27.

[26] R. Jensen, P.-L. Lions \& P. E. Souganidis, A uniqueness result for viscosity solutions of second order fully nonlinear partial differential equations, Proc. Amer. Math. Soc. 102 (1988) 975-978.

[27] A. U. Kennington, Power concavity and boundary value problems, Indiana Univ. Math. J. 34 (1983) 687-704.

[28] _ _ Power concavity of solutions of Dirichlet problems, Proc. Centre Math. Anal. Austral. Nat. Univ. 8 (1984) 133-136.

[29] N. J. Korevaar, Convex solutions to nonlinear elliptic and parabolic boundary value problems, Indiana Univ. Math. J. 32 (1983) 603-614.

[30] N. V. Krylov, Nonlinear elliptic and parabolic equations of second order, Reidel, Dordrecht, 1987.

[31] O. A. Ladyzhenskaja, V. A. Solonnikov \& N. N. Ural'tseva, Linear and quasilinear equations of parabolic type, Amer. Math. Soc., Providence, RI, 1968.

[32] P.-L. Lions, Optimal control of diffusion processes and Hamilton-Jacobi-Bellman equations. I, Comm. Partial Differential Equations 8 (1983) 1101-1134.

[33] S. Osher \& J. A. Sethian, Fronts propagating with curvature dependent speed: algorithms based on Hamilton-Jacobi formulations, J. Computational Phys. 79 (1988) 12-49.

[34] H. Parks \& W. Ziemer, Jacobi fields and regularity of functions of least gradient, Ann. Scuola Norm. Sup. Pisa Cl. Sci. (4) 11 (1984) 505-527. 
[35] J. A. Sethian, Recent numerical algorithms for hypersurfaces moving with curvaturedependent speed: Hamilton-Jacobi equations and conservation laws, J. Differential Geometry 31 (1990) 131-161.

[36] N. S. Trudinger, The Dirichlet problem for the prescribed curvature equations, preprint, 1989.

UNIVERSity OF CALIForNia, BERKeley

UNIVERSITY OF MASSACHUSETTS 
